\title{
Die Staatenzentriertheit des Menschenrechtsschutzes als Grenze der Begrenzung von (Hoheits)gewalt ${ }^{*}$
}

\begin{abstract}
Summary
The breach of human-rights protected content by non-governmental actors accounts for one of the contemporary major challenges of human rights protection, the complexity of the exercised sovereign public authority and the advancing deterritorialization, together with the simultaneous ongoing state-centricity of the human rights regime. The state-centricity of human rights protection is to be seen therein that human rights regimes are still geared to limit sovereign public authority in the "conventional" legal expression of states. This condition brings about, as will be shown in the present contribution, that restrictions are set to sovereign public authority by human right. Complex execution of sovereign public authority requires complex answers. The international legal order currently finds itself in a transitional phase. The described challenges have been recognized, satisfactory solutions have so far not yet been found. Regardless of how the international legal order might look like in detail, flexibility and differentiation are necessary in times in which the rigid concept of state sovereignty no longer reflects reality as a point of departure in its limitation of violence against individuals.
\end{abstract}

\section{Resumé}

Parmi les grands défis actuels de la protection des droits de l'homme figurent notamment la violation par des acteurs non étatiques de contenus protégés par les droits de l'homme, la complexité de l'exercice de l'autorité de l'État et la rapidité du processus de déterritorialisation, tandis que les régimes des droits de l'homme continuent à être centralisés au sein des États. Cette centralisation de la protection des droits de l'homme au sein des États s'explique par le fait que les régimes des droits de l'homme visent toujours à restreindre l'autorité de l'État dans le sens «traditionnel » que lui donne le droit public. Comme l'expose le présent article, cela permet de limiter la restriction de l'autorité de l'État par les droits de l'homme. La complexité de l'exercice de l'autorité de l'État demande des réponses complexes. Le système de droit international traverse actuellement une phase de transition. Si les défis précités ont bien été identifiés, aucune solution satisfaisante n'a toutefois encore été trouvée. Quelle que soit la réponse détaillée qu'apportera le système de droit international, la flexibilité et la différenciation sont incontournables à une époque à laquelle le concept rigide d'autorité de l'État, censé appuyer la limitation de la violence contre les individus, ne correspond plus à la réalité.

* Anna Fontaine ist wissenschaftliche Mitarbeiterin an der Professur für Öffentliches Recht, Völker- und Europarecht von Prof. Dr. Dr. Rainer Hofmann an der Goethe Universität Frankfurt am Main. 


\section{A. Einleitung}

Die rechtliche Begrenzung der Ausübung von Hoheitsgewalt gegenüber Individuen erfolgt durch die Grund- und Menschenrechte. Es stehen hierfür nationale Grundrechte und völkerrechtliche, dem Menschenrechtsschutz dienende Normen, die in universell oder nur regional geltenden Instrumenten zum Menschenrechtsschutz enthalten sind, zur Verfügung. Zudem steht in bewaffneten Konflikten das humanitäre Völkerrecht bereit.

Menschenrechtsregime sind, wie das Völkerrecht im Allgemeinen, historisch staatenzentriert. ${ }^{1}$ Ihre Aufgabe bestand bislang darin, Hoheitsgewalt im „herkömmlichen“ staatenrechtlichen Sinn zu begrenzen. Dies ist im Staatsgebiet die Tätigkeit als originäre, ursprüngliche unwiderstehliche Herrschaftsmacht. ${ }^{2}$ Hoheitsgewalt wird durch den Staat ausgeübt, indem der Gesetzgeber verbindliche Regelungen trifft, die vollziehende Gewalt verbindlich gebietet und verbietet und die rechtsprechende Gewalt autoritativ entscheidet. $^{3}$

Wir leben heute jedoch in Zeiten „,komplexer Hoheitsgewalt“. ${ }^{4}$ Staatsgewalt ist nicht mehr nur auf das Territorium eines Staates begrenzt und existiert dort gleichzeitig als einzige Staatsgewalt, die sich ihre Herrschaftsberechtigung mit niemandem teilen muss. ${ }^{5}$ Bestehende Instrumente zur Begrenzung von Hoheitsgewalt, ausgehend von der Prämisse, dass sie der Begrenzung von Hoheitsgewalt im „herkömmlichen“ staatenrechtlichen Sinn dienen, erfahren ihre Grenzen. Der folgende Beitrag möchte aufzeigen, welche Entwicklungen das ,herkömmliche“ staatenrechtliche Verständnis von Hoheitsgewalt und die Staatenzentriertheit des Menschenrechtsschutzes in Frage stellen und inwieweit sich Grenzen der Begrenzung von Hoheitsgewalt aus der vorherrschenden und noch genauer zu betrachtenden Staatenzentriertheit des Menschenrechtsschutzes ergeben.

1 s. z.B. Shelton, Protecting Human Rights in a Globalized World, BCICLR 2002, 273; Mégret/ Hofmann, The UN as a Human Rights Violator? Some Reflection on the United Nations Changing Human Rights Responsibilities, HRQ 2003, 314, 320; Janik, Die Bindung internationaler Organisationen an internationale Menschenrechtsstandards, 2012, 14 m.w.N.

2 Stern, Das Staatsrecht der Bundesrepublik Deutschland. Band II, 1980, 533.

3 Hesse, Grundzüge des Verfassungsrechts der BRD, 1999, § 13, Rn. 491.

4 Peters, Die Anwendbarkeit der EMRK in Zeiten komplexer Hoheitsgewalt und das Prinzip der Grundrechtstoleranz, AVR 2010, 1.

5 Feinäugle, Hoheitsgewalt im Völkerrecht: Das 1267-Sanktionsregime der UN und seine rechtliche Fassung, 2011, 23; Cassese, Administrative Law without a State?, The Challenge of Global Regulation, NYUJILP 2005, 663, 673, für den die zentrale Rolle des Staates für den Begriff der Hoheitsgewalt eine optische Illusion geworden ist; Grimm, Die Verfassung im Prozess der Entstaatlichung, in: Brenner, et al. (Hrsg.), Der Staat des Grundgesetzes - Kontinuität und Wandel, FS Badura, 2004, 145, 155. 


\section{B. Die Infragestellung des ,herkömmlichen“ staatenrechtlichen und staa- tenzentrierten Verständnisses von Hoheitsgewalt beim Individualrechts- schutz}

Die Verletzung von menschenrechtlich gewährten Inhalten erfolgt, wie anhand von Beispielen zu zeigen ist, auch durch Tätigkeiten, die außerhalb der „herkömmlichen“ staatenrechtlichen Definition von Hoheitsgewalt liegen.

\section{Gewaltausübung gegen Individuen durch nichtstaatliche Akteure}

Es entspricht nicht (mehr) der Wirklichkeit des Völkerrechts, dass nur Staaten die Fähigkeiten haben, die Rechte von Individuen zu beeinträchtigen. Als weitere Akteure, die potenziell menschenrechtlich geschützte Inhalte gefährden, sind internationale Organisationen und „Private“ zu nennen.

\section{Gewaltausübung gegen Individuen durch internationale Organisationen}

Lange Zeit bestand Einigkeit darüber, dass Staaten die einzigen Völkerrechtssubjekte sind und folglich nur sie Träger von völkerrechtlichen Pflichten und Rechten sein können. Es hat jedoch eine Entwicklung vom klassischen Verständnis des Staates mit seiner nach außen abgeschlossenen Souveränität hin zu einer Tätigkeit von Akteuren auf völkerrechtlicher Ebene zur Erfüllung von Aufgaben, die über das Nationale hinausgehen, stattgefunden. ${ }^{6}$ Sachbereiche wie Wirtschaft, Umwelt und Sicherheit können heute nicht mehr rein national befriedigend geregelt werden. ${ }^{7}$ Diese Entwicklung wird mit den Begriffen Entstaatlichung, ${ }^{8}$ Internationalisierung, ${ }^{9}$ bzw. Globalisierung, ${ }^{10}$ beschrieben, wobei Unterschiede insoweit bestehen als die oben beschriebenen Phänomene aus verschiedenen Perspektiven betrachtet werden. ${ }^{11}$ Internationale Organisationen haben von den Staaten Kompetenzen übertragen bekommen, die es ihnen erlauben in diesen internationalisierten Sachbereichen normsetzend tätig werden zu können. ${ }^{12}$ Mittlerweile anerkannt ist, dass internationale Organisationen partielle Völkerrechtssubjekte sein

6 Vgl. Slaughter, A New World Order, 2004, 4; Feinäugle, (Fn. 5), 24.

7 Schorkopf/Walter, Elements of Constitutionalization: Multilevel Structures of Human Rights Protection in General International and WTO-Law, GLJ 2003, $1359 \mathrm{ff}$.

8 Vgl. z.B. Grimm, (Fn. 5), 145 und 156 ff.; Cassese, (Fn. 5), 673.

9 Vgl. z.B. Fassbender, „Wo aber Gefahr ist, wächst das Rettende auch?“ Die Internationalisierung von Risiken und die Entwicklung des völkerrechtlichen Katastrophenschutzes, KritV 2005, 375 ff.; Bogdandy, Das deutsche Staatshaftungsrecht vor der Herausforderung der Internationalisierung, AöR, 1997, $268 \mathrm{ff}$.

10 Vgl. Ruffert, Die Globalisierung als Herausforderung an das Öffentliche Recht, 2004, 16 ff. m.w.N.

11 Schorkopf/Walter, (Fn. 7), $1359 \mathrm{ff}$.

12 Feinäugle, (Fn. 5), 26. 
können. ${ }^{13}$ Korrelat der Rechtspersönlichkeit internationaler Organisationen im Völkerrecht ist die Verantwortlichkeit für eigenes völkerrechtswidriges Handeln. ${ }^{14}$

Der Bedarf nach einer Bindung internationaler Organisationen an Menschenrechtsstandards ist damit zu begründen, dass sie die Fähigkeit besitzen, die Rechte von Individuen zu beeinträchtigen, ${ }^{15}$ und Individuen in ähnlicher Weise determinieren können, wie es ursprünglich Staaten vorbehalten war. ${ }^{16}$ Im Schrifttum hierfür angeführte Beispiele sind unter anderem die Handlungen des Sicherheitsrates im Rahmen seiner Befugnisse unter Kapitel VII UNCh (militärische Maßnahmen, klassische wirtschaftliche Sanktionen und targeted sanctions, die Errichtung Internationaler Straftribunale), ${ }^{17}$ das Handeln der United Nations Interim Administration Mission in Kosovo (UNMIK) im Kosovo, bei deren Verwaltungshandeln im Rahmen ihres Mandates zahlreiche Berührungspunkte mit international geschützten Menschenrechten gesehen werden, ${ }^{18}$ das Handeln von Weltbank und Internationalem Währungsfonds, wenn sie z.B. Kredite an Staaten vergeben, in denen systematische Menschenrechtsverletzungen stattfinden, ${ }^{19}$ sowie die Festlegung des Flüchtlingsstatus durch den Hohen Flüchtlingskommissar der Vereinten Nationen (UNHCR). ${ }^{20}$ Außerdem ist das Handeln der Europäischen Union (EU) -durch ihre Organe oder die Organe ihrer Mitgliedstaaten- zu nennen, die selbst erkannt hat, dass sie als supranationale Organisation die Rechte von Individuen verletzen kann und sich deshalb nicht nur eine Selbstbindung in Form der Bindung an die Unionsgrundrechte (Art. 6 AEUV Abs. 1, 3 AEUV) auferlegt hat, sondern durch den geplanten Beitritt zur Europäischen Menschenrechtskonvention (EMRK) auch eine Bindung an einen externen Menschenrechtskatalog anstrebt (Art. 6 Abs. 2 EUV) und bereit ist, sich der Rechtsprechung eines externen Gerichts (Europäischer Gerichtshof für Menschenrechte (EGMR)) zu unterwerfen.

\section{Gewaltausübung gegen Individuen durch „Private“}

Als Beispiel für Eingriffe in die Rechte von Individuen durch „Private“ kann die Gewaltausübung durch Aufständische und inoffizielle Machthaber genannt werden. ${ }^{21}$ Fer-

13 Partiell, weil sie zur Teilnahme am völkerrechtlichen Rechtsverkehr nur befähigt sind, soweit sie hierfür in ihrer Satzung von ihren Mitgliedern Befugnisse übertragen bekommen haben; Vgl. Dahm/Delbrück/Wolfrum, Völkerrecht, Band I/1, 1988, § 2, 22.

14 vgl. z.B. Ruffert/ Walter, Institutionalisiertes Völkerrecht, Das Recht internationaler Organisationen und seine wichtigsten Anwendungsfelder, 2009, § 7, Rn. 215.

15 Mégret/Hofmann, (Fn. 1), 314, 323 ff.; Janik, (Fn. 1), 28.

16 Janik, (Fn. 1), 18.

17 Ibid., 82 ff.; 86 ff.; 102.

18 Moos, Individualrechtsschutz gegen menschenrechtswidrige hoheitliche Maßnahmen von Übergangsverwaltungen der VN am Beispiel der UNMIK in Kosovo, 2013, $55 \mathrm{ff}$.

19 Vgl. Janik, (Fn. 1), 109; Hofmann, Die Rechtskontrolle von Organen der Staatengemeinschaft, in: ders. et al. (Hrsg.), Die Rechtskontrolle von Organen der Staatengemeinschaft, Vielfalt der Gerichte- Einheit des Prozessrechts?, Berichte der Deutschen Gesellschaft für Völkerrecht, 2007, 1, $16 \mathrm{f}$.

20 Feinäugle, (Fn. 5), $40 \mathrm{f}$.

21 Thürer, Herkules und die Herausforderungen des modernen Menschenrechtsschutzes, in: ders., Völkerrecht als Fortschritt und Chance, Grundidee Gerechtigkeit, Band 2, 2009, 585 , 593. 
ner werden zunehmend Aufgaben, die vormals durch den Staat wahrgenommen wurden, und denen ein menschenrechtsverletzendes Potenzial inne wohnt, in den privaten Bereich verlagert (Privatisierung). ${ }^{22}$ Auf völkerrechtlicher Ebene ist insoweit insbesondere der Einsatz privater Sicherheitsfirmen von Interesse, die mittlerweile eine ganze Bandbreite von Dienstleistungen anbieten, die von Tätigkeiten im Bereich der Logistik (Transport, Verpflegung, Instandhaltung militärischer Liegenschaften, Wartung der Fahrzeugflotte etc.) über Informations- und Nachrichtendienste, die Sicherung von Liegenschaften, Konvois, individueller Personen bis zu Kampfeinsätzen reichen. ${ }^{23}$ Aufgaben, die vormals vom Staat wahrgenommen wurden, für die teilweise sogar ein staatliches Monopol bestand, beziehungsweise die zumindest von der Öffentlichkeit als staatliche Aufgaben wahrgenommen wurden, sind somit auf „Private“ übertragen worden. Beispiel hierfür ist der Einsatz privater Sicherheitskräfte auf Handelsschiffen, die diese Schiffe vor Angriffen von Piraten schützen sollen und der zumindest teilweise von Staaten angeordnet ist, ${ }^{24}$ obwohl jedenfalls das Seerechtsübereinkommen der Vereinten Nationen ein staatliches Monopol der Pirateriebekämpfung festlegt. ${ }^{25}$ Außerdem stellt die Übertragung von militärischen Funktionen auf private Militärfirmen, wie z.B. durch die US-Regierung im Irak geschehen, die staatliche Monopolisierung von Gewalt und die Verstaatlichung des Krieges in Frage. ${ }^{26}$ Insbesondere die Militärfirma Blackwater/ Xe Services hat traurige Berühmtheit erlangt, nachdem dessen Personal ohne Anlass siebzehn Zivilisten auf dem belebten Nisor Platz in Bagdad tötete. ${ }^{27}$

Gedacht werden kann in diesem Zusammenhang aber auch an Medienkonzerne oder an mächtige multinationale Unternehmen, die das Leben der Menschen in der „Dritten Welt" oft dominieren. ${ }^{28}$ Diese gesellschaftlichen Machtgebilde können für die Freiheit von Individuen viel bedrohlicher sein als dies die Herrschaft des Staates von heute ist oder in der Vergangenheit gewesen war. ${ }^{29}$

22 vgl. z.B. Brehme, Privatisierung und Regulierung der öffentlichen Wasserversorgung, 2010, 15 .

23 Vgl. z.B. Schaller, Schutz transnationaler Unternehmen in Konfliktregionen. Rechtliche Betrachtung zum Einkauf privater Sicherheit, S+F 2006, 78, 79.; ausführliche Darstellung der verschiedene Typen privater Sicherheit-und Militärunternehmen s. Kümmel, Die Privatisierung der Sicherheit. Private Sicherheits- und Militärunternehmen in den internationalen Beziehungen, ZIB 2005, 141, $146 \mathrm{ff}$.

24 z.B. Liberia empfahl spezifische Dienstleister mit denen bereits gewisse Erfahrungswerte bestanden, s. Salomon/tho Pesch, Das Zulassungsregime für bewaffnete Sicherheitsdienste auf Handelsschiffen, DÖV 2013, 760, 761; Salomon/ tho Pesch, Zertifizierung bewaffneter Sicherheitskräfte auf deutschen Handelsschiffen und Staatshaftung, NordÖR 2012, 65, 70.

25 Von Arnauld, Die moderne Piraterie und das Völkerrecht, AVR 2009, 454, 464 ff.; Trésoret, Seepiraterie, völkerrechtliche, europarechtliche und verfassungsrechtliche Rahmenbedingungen der Auslandsentsendung deutscher Streitkräfte zur Bekämpfung der Seeräuberei, 2011, 363.

26 Lehnardt, Privat Militärfirmen und völkerrechtliche Verantwortlichkeit, 2011, 1; Chestermann/Lehnardt, Introduction, in: dies.(eds.), From mercenaries to market, The Rise and Regulation of Private Military Companies, 2007, 1.

27 Spiegel Online, US-Militärs belasten Blackwater-Söldner, 5.10.2007.

28 Thürer, (Fn. 21), 594.

29 Ibid. 


\section{Entterritorialisierung}

Staatsgewalt ist nicht mehr nur auf das Territorium eines Staates begrenzt und existiert dort gleichzeitig als einzige Staatsgewalt, die sich ihre Herrschaftsberechtigung mit niemandem teilen muss. ${ }^{30}$ Zum eine greifen Staaten nicht nur auf ihrem Staatsgebiet in die Freiheitssphäre von Menschen ein. Diverse militärische und zivile Auslandseinsätze, Drohneneinsätze auf fremdem Territorium, aber auch die Ausspähaktionen der Daten von Personen, die sich im Ausland befinden, der Amerikaner und der Briten, die unter den Stichworten „PRISM“ und ,Tempora “ bekannt geworden sind, zeigen, dass Verletzungen der Menschenrechte durch Staaten auch außerhalb des eigenen Territoriums geschehen. Zum anderen treffen überstaatliche Einheiten oder Institutionen Entscheidungen, welche auf dem Territorium der Staaten Geltung beanspruchen (Entstaatlichung). ${ }^{31}$

Außerdem verfügen internationale Organisationen und „Private“ als weitere Akteure, die Gewalt gegen Individuen ausüben können, über keine Staatsgebiete.

\section{Staatenzentriertheit als Grenze der Begrenzung von (Hoheits)gewalt}

$\mathrm{Zu}$ betrachten ist, inwieweit den oben beschriebenen Entwicklungen Rechnung getragen wird und Verletzungen menschenrechtlicher Inhalte durch Tätigkeiten, die außerhalb der „herkömmlichen“ staatenzentrierten Definition von Hoheitsgewalt liegen, durch die Menschenrechte Grenzen gesetzt werden.

\section{Die Bindung nichtstaatlicher Akteure an menschenrechtliche Standards}

Menschenrechtliche Verpflichtungen stellen das Individuum in den Mittelpunkt. ${ }^{32}$ Nur in Einzelfällen ist es möglich, dass ein Rechtssatz inhaltlich auf spezifische staatliche Qualitäten abstellt, weshalb dieser unanwendbar auf internationale Organisationen ${ }^{33}$ oder „Private“ wäre. Der Inhalt menschenrechtlicher Verpflichtungen steht einer Bindung anderer Akteure als Staaten deshalb grundsätzlich nicht entgegen.

30 Feinäugle, (Fn. 5), 23; Cassese, (Fn. 5), 663, 674.

31 Grimm, (Fn. 5), 145, 156 f.

32 vgl. z.B. Fassbender, Idee und Anspruch der Menschenrechte im Völkerrecht, APuZ 2008, Heft 46, 3, 7; Janik, (Fn. 1), 14.

33 Bothe, Streitkräfte internationaler Organisationen zugleich ein Beitrag zu völkerrechtlichen Grundfragen der Anwesenheit fremder Truppe, 1968, 14; Kondoch, Human rights law and UN peace operations in Post-conflict situations, in: White/Klaasen (Hrsg.), The UN, human rights and post conflict situations, 2005, 19, 36. 


\section{Internationale Organisationen}

a) Völkervertragliche Bindung internationaler Organisationen an Menschenrechtsstandards

Die primär staatliche Verpflichtung aus menschenrechtlichen Verträgen folgt deshalb meist nicht aus den einzelnen menschenrechtlichen Verpflichtungen selbst, sondern aus dem Teilnehmerkreis des Menschenrechtsvertrages und aus der passiven Parteifähigkeit bei Bestehen eines rechtlichen Überwachungssystems. ${ }^{34}$ Die Lösbarkeit der Probleme, die entstehen, wenn internationale Organisationen an Verträge gebunden werden sollen, die ursprünglich nur auf Staaten ausgerichtet sind, zeigt der am 4. Juli 2013 veröffentlichte Entwurf des Steering Comittee for Human Rights (CDDH) über einen Beitritt der EU zur EMRK. ${ }^{35}$ Ferner hat die EU bereits am 23. Oktober 2010 die UN-Behindertenrechtskonvention als gemischtes Abkommen ratifiziert. ${ }^{36}$ Der Beitritt der EU zur EMRK, als einem umfassenden Menschenrechtskatalog, ist bislang jedoch noch nicht vollzogen und ein weiterer Beitritt einer internationalen Organisation zu menschenrechtlichen Verträgen ist bislang nicht geplant. Im Schrifttum wird deshalb teilweise versucht, eine Bindung internationaler Organisationen an menschenrechtliche Verträge damit zu begründen, dass sich die Mitgliedstaaten durch die Gründung einer internationalen Organisation nicht von ihren völkerrechtlichen Verpflichtungen befreien könnten und sie diese deshalb bei Schaffung der internationalen Organisation zusammen mit den entsprechenden Kompetenzen auf sie übertragen hätten, weshalb die internationalen Organisationen automatisch aus den völkerrechtlichen Verträgen ihrer Mitglieder verpflichtet worden seien. ${ }^{37}$ Außerdem wird eine weitreichende Bindung internationaler Organisationen an menschenrechtliche Standards im Schrifttum damit zu begründen versucht, dass, wenn diese die Achtung oder die Förderung der Achtung der Menschenrechte in ihren Gründungsverträgen als Ziele aufführten, sich eine Bindung aus den Gründungsverträgen selbst ergebe. ${ }^{38}$ Hierauf stützt sich auch eine weitere Ansicht, die aus dem Grundsatz von Treu und Glauben, dem Estoppel-Prinzip, eine Bindung inter-

34 Janik, (Fn. 1), $14 \mathrm{f}$.

35 Fifth Negotiation meeting between the CDDH AD Hoc Negotiation Group and the European Commission on the Accession of the European Union to the European Convention on Human Rights, Final Report of the CDDH, 3. April -5 April 2013, 47+1(2013)008rev 2, abrufbar unter: http://www.coe.int/t/dghl/standardsetting/hrpolicy/Accession/Meeting_reports/ 47_1\%282013\%29008rev 2_EN.pdf [30.7.2014].

36 s. Antwort der Bundesregierung auf die Kleine Anfrage der Abgeordneten Dr. Ilja Seifert, Diana Golze, Heidrun Dittrich, weiterer Abgeordneter und der Fraktion DIE LINKE.- Drucksache 17/10563 -, BT-Drucksache 17/10701, 1.

37 Osteneck, Die Umsetzung von UN-Wirtschaftssanktionen durch die EG: völker- und europarechtliche Rahmenbedingungen für ein Tätigwerden der EG im Bereich von UN-Wirtschaftssanktionsregimen unter besonderer Berücksichtigung der Umsetzungspraxis der EGOrgane, 2004, 222 ff.; s. für weitere Theorien, die eine Bindung internationaler Organisationen an menschenrechtliche Verträge begründen wollen, Janik, (Fn. 1), $389 \mathrm{ff}$.

38 vgl. z.B. Für die Menschenrechtsbindung des Sicherheitsrates: Aus Art. 24 Abs. 2 i.V.m. Art. 1 Abs. 3 UNCh Kotzur, Entscheiden(d) für die Welt - zur Rationalität und Legitimität der Entscheidungen des UN-Sicherheitsrates, in: JöR, 2007, 23, 36; aus Art. 1 Abs. 3 UNCh, wenn die UN jursidiction über Individuen ausübt, Fassbender, Targeted Sanctions Imposed by the UN Security Council and Due Process Rights, IOLR 3, 2006, 437, 473. 
nationaler Organisationen an menschenrechtliche Standards begründet. ${ }^{39}$ Wenn eine internationale Organisation einen Beitrag zur Entwicklung des internationalen Menschenrechtsschutzes leiste, würden sie die berechtigte Erwartung wecken, dass sie sich selbst an die Menschenrechte halte. ${ }^{40}$

b) Bindung internationaler Organisationen an Menschenrechtsstandards abseits der völkervertraglichen Bindung?

Im Schrifttum besteht weitestgehend Einigkeit darüber, dass internationale Organisationen an die menschenrechtlichen Standards, die zwingendes Völkerrecht (ius cogens) bilden, gebunden sind. ${ }^{41}$ Auch wenn kein Konsens über den Inhalt eines völkerrechtlichen ius cogens besteht (überwiegend werden Völkermord, das Gewaltverbot, das Folterverbot, ${ }^{42}$ das Verbot des Menschenhandels, der Rassendiskriminierung sowie der Sklaverei als Inhalt der ius cogens anerkannt), ${ }^{43}$ lässt sich sagen, dass die Menschenrechte als solche nicht Inhalt der ius cogens sind, weshalb sich aus ihnen keine weitreichende Bindung an menschenrechtliche Standards begründen lässt.

Ferner wird angenommen, dass internationale Organisationen an völkergewohnheitsrechtliche Menschenrechtsstandards gebunden seien, ${ }^{44}$ da das Völkergewohnheitsrecht Wirkung für alle Völkerrechtssubjekte beanspruche. ${ }^{45}$ Bislang ist jedoch nur bei einigen wenigen Menschenrechten der gewohnheitsrechtliche Charakter anerkannt, ${ }^{46}$ während bei der Mehrheit der gewohnheitsrechtliche Status ungeklärt und umstritten ist. ${ }^{47} \mathrm{Be}$ troffen von dieser Unklarheit sind nicht nur völkerrechtlich noch „unterentwickelte“ Rechte, wie der Schutz der Privatsphäre, der Bewegungs- und Freizügigkeit, das Persönlichkeitsrecht, der Eigentumsschutz oder die Justizgrundrechte, sondern auch elementare Rechte, wie das Recht auf Leben und körperliche Unversehrtheit oder der Schutz vor willkürlicher Freiheitsentziehung, sowie die Fragen nach den Eingriffsvoraussetzungen und einzuhaltender Mindeststandards bei Einschränkungen und Schutzpflichten. $^{48}$

Weitreichender ist die Bindung internationaler Organisationen an menschenrechtliche Standards, die im Schrifttum damit begründet wird, dass diese an die Verfassungsprinzipien des Menschenrechtsschutzes und der Rechtsstaatlichkeit, die allgemeine Rechts-

39 Vgl. z.B. de Wet, The Chapter VII Powers of the United Nations Security Council, 2004, 195.

40 Ibid., $199 \mathrm{f}$.

41 Vgl. z.B. Moos, (Fn. 18), 262; Janik, (Fn. 1), 529 m.w.N.; Hofmann, (Fn. 19), 12 ff.

42 s. Moos, (Fn. 18), 249 m.w.N.

43 s. Oeter, Ius cogens und der Schutz der Menschenrechte, in: Breitenmoser, et al. (Hrsg.), Menschenrechte, Demokratie und Rechtsstaat, FS Wildhaber, 499, 506, $512 \mathrm{ff}$.

44 z. B. Reinisch, Developing Human Rights and Humanitarian Law Accountability of the Security Council for the Imposition of Economic Sanctions, AJIL 95, 2001, 851, 858; Hofmann, (Fn. 19), $16 \mathrm{ff}$.

45 Reinisch, (Fn. 44), 858; Moos, (Fn. 18), 228.

46 Herdegen, Völkerrecht, 2012, § 47 Rn. 3; Moos, (Fn. 18), 240.

47 s. hierzu ausführlich, auch zu den einzelnen Rechten, Moos, (Fn. 18), $240 \mathrm{ff}$.

48 Ibid., 253. 
grundsätze darstellten (Art. 38 Abs. 1 lit. c IGH-Statut) und die aus nationalen und internationalen Quellen abgeleitet werden, gebunden seien. ${ }^{49}$

\section{Individuen}

In der jüngsten Völkerrechtsentwicklung wurde anerkannt, dass auch das Individuum eigene Rechte (Menschenrechte) und Pflichten haben kann. Für schwerwiegende Verletzungen völkerrechtlicher Pflichten kann es deshalb auch persönlich zur Verantwortung gezogen werden. ${ }^{50}$ Die Sanktionierung schwerwiegender Verletzungen des Völkerrechts erfolgt durch das Völkerstrafrecht „,der Gesamtheit der Normen, die ein bestimmtes individuell vorwerfbares Verhalten bei Androhung von Strafe verbieten, sich aus Völkerrechtsquellen speisen und unmittelbar für das Individuum gelten ". 51 Anerkannte Völkerrechtsverbrechen sind der Völkermord (Art. 6 IStGHSt), Verbrechen gegen die Menschlichkeit (Art. 7 IStGHSt), Kriegsverbrechen (Art. 8 IStGHSt) und das Verbrechen der Aggression (Art. 5 Abs. 1, 2; Art. 8 bis IStGHSt). ${ }^{52}$ Viele schwere Menschenrechtsverletzungen lösen die Verantwortlichkeit „Privater“ jedoch nicht aus und jenseits des Strafrechts bestehen nur vereinzelte Ansätze für eine Verantwortlichkeit von Individuen. ${ }^{53} \mathrm{Zu}$ nennen sind hier etwa die, allerdings noch in ihren Anfängen begriffenen, Bemühungen, transnationale Unternehmen durch internationale Standards in die Pflicht zu nehmen. ${ }^{54}$ Eine umfassende direkte Bindung „Privater“ an menschenrechtliche Standards besteht nicht.

Als nicht völkerrechtliche Regulierungsansätze mit dem Ziel, die Verwirklichung und die Durchsetzung des Völkerrechts und der Menschenrechtsübereinkommen zu gewährleisten, sind aber bestehende Mechanismen der Selbstregulierung „Privater“ und die innerstaatliche Haftung als Instrumente der mittelbaren Durchsetzung des Völker-

49 vgl. z.B. Ibid., 254 ff. und 263 ff.; Simma/Alston,, The Source of Human Rights Law: Custom, Jus Cogens, and General Principles, 12 Australian YBIL, 1989, 82, 102 ff.; Kadelbach/Kleinlein, International Law - a Constitution for Mankind? An Attempt at a Re-appraisal with an Analysis of Constitutional Principles, GYIL 50 (2007), 303, 342; Janik, (Fn. 1), $488 \mathrm{ff}$.

50 Herdegen, (Fn. 46), § 7 Rn. 5.

51 Christoph, Internationales Strafrecht, Strafanwendungsrecht, Völkerstrafrecht, Europäisches Strafrecht, 2011, 4 Rn. 3.

52 Safferling, (Fn. 51), § 4 Rn. 8.

53 z.B. die Haftung von privaten Vertragsnehmern für Schäden beim Tiefseebergbau, vgl. Annex III, Art. 22 United Nations Convention on the Law of the Sea, 1982. Im Bereich der Kernenergie Haftung der Betreiber für Schäden nach nationalem Recht: vgl. z.B. Art. 3 OECD Paris Convention on Third Party Liability in the Field of Nuclear Energy, 1960; ebenso Vienna Convention on Civil Liability for Nuclear Damage, 1963. Siehe ausführlich mit weiteren Beispielen Tomuschat, The Responsibility of Other Entities: Private Individuals, in: Crawford et al. (Hrsg.), The Law of International Responsibility, 2010, 317, $323 \mathrm{ff}$.

54 s. hierzu ausführlicher Shelton, (Fn. 1), 314 und z.B. Commission on Human Rights, Reports of the Working Group on the Effects of the Working Methods and Activities of Transnational Corporations on Human Rights, abrufbar unter: http://www.ohchr.org/EN/Issues/Business/ Pages/Reports.aspx [30.7.2014]. 
rechts zu nennen. ${ }^{55}$ Beispielsweise haben sich private Sicherheitsfirmen als Antwort auf die Vorwürfe, dass sie Menschenrechte und Arbeitnehmerrechte verletzen, vielfach freiwillige Verhaltenskodizes (codes of conduct) auferlegt, die völkerrechtliche Pflichten für den Privatsektor „übersetzen“ sollen. ${ }^{56}$ Als ein Instrument der mittelbaren Durchsetzung des Völkerrechts gegenüber „Privaten“ stellt sich zudem der amerikanische Alien Tort Claims Act (ATCA) dar. ${ }^{57}$ Er begründet die Zuständigkeit von USBundesgerichte für die Verletzung von Völkerrecht und wurde in den letzten Jahren verstärkt als Grundlage für Klagen wegen Menschenrechtsverletzungen durch „Private“ genutzt. ${ }^{58}$ Allerdings hat die jüngst (17. August 2013) ergangene Entscheidung des Supreme Courts oft the United States im Kiobel-Verfahren ${ }^{59}$ den Anwendungsbereich für Klagen wegen Menschenrechtsverletzungen nunmehr beschränkt. ${ }^{60}$ Der Supreme Court statuierte, dass die Vermutung der Nichtanwendbarkeit der ATCA außerhalb des Territoriums der USA, nur widerlegt werden könne, wenn Unternehmenshandlungen einen Bezug zu den USA aufweisen, der über eine bloße Unternehmenspräsenz hinausgehe. ${ }^{61}$

Wieder auf völkerrechtlicher Ebene wird oftmals die Verantwortung der Staaten für durch „Private“ erfolgte Menschenrechtsverletzungen begründet. Zum einen erfolgt dies über die Zurechnung des Verhaltens „Privater“ zum Staat, zum anderen werden den Staaten „Schutzpflichten“ für die Verwirklichung von Menschenrechten auch im gesellschaftlichen Raum auferlegt. ${ }^{62}$

\section{Zwischenfazit}

Der Bedarf für eine eigene Bindung internationaler Organisationen an menschenrechtliche Standards wird, wie die vielfältigen im Schrifttum existierenden Versuche eine solche Bindung zu begründen zeigen, allgemeinhin gesehen. Bislang ist jedoch nur die EU der UN-Behindertenrechtskonvention, als einem menschenrechtlichen Vertrag, beigetreten und die meisten menschenrechtlichen Verträge stehen weiterhin nur Staaten zur Ratifikation offen. Die Konstruktionen einer vertraglichen Bindung internationaler Organisationen an menschenrechtliche Standards, die eine umfassende Bindung an menschenrechtliche Standards zur Folge hätten, verstoßen gegen den Grundsatz des Verbotes der Drittwirkung von Verträgen (pacta tertiis-Regel). ${ }^{63}$ Zudem lässt sich auch aus den Gründungsverträgen internationaler Organisationen - außer sie sehen, wie die

55 s. ausführlich Lehnardt, (Rn. 26), 44 ff.; für eine Unterscheidung bestehender codes of conduct s. Hennings, Über das Verhältnis von Multinationalen Unternehmen zu Menschenrechten: Eine Bestandsaufnahem aus juristischer Perspektive, 2009, 55.

56 s. z.B. Voluntary Principles on Security and Human Rights, abrufbar unter: http://www.voluntaryprinciples.org/ [30.7.2014].

57 s. Seibert-Fohr/Wolfrum, Die einzelstaatliche Durchsetzung völkerrechtlicher Mindeststandards gegenüber transnationalen Unternehmen, AVR 43, 2005, 153-186.

58 Lehnardt, (Rn. 26), 51.

59 Kiobel v. Royal Dutch Petrol. Co., 133 S. Ct. 1669 (2013).

60 Vgl. Fox, Hazel/ Webb, Philippa, The Law of State Immunity, Third Edition, 2013, 275.

61 Kiobel v. Royal Dutch Petrol. Co., 133 S. Ct. 1669.

62 Thürer, (Rn. 21), 594.

63 Janik, (Fn. 1), 423. 
der EU ihre Bindung an menschenrechtliche Standards ausdrücklich vor (Art. 6 EUV) - keine Bindung einer internationalen Organisation an menschenrechtliche Standards erreichen. Der Wortlaut der Vorschriften der Gründungsverträge steht einer solchen Interpretation (meist) entgegen. ${ }^{64}$ Eine umfassende Bindung internationaler Organisationen an menschenrechtliche Standards lässt sich jedoch damit begründen, dass die Verfassungsprinzipien des Menschenrechtsschutzes und der Rechtsstaatlichkeit allgemeine Rechtsgrundsätze darstellen, an die auch internationale Organisationen gebunden sind. ${ }^{65}$ Die praktische Bedeutung dieser so begründeten Bindung an menschenrechtliche Standards ist bislang jedoch gering. Klagen gegen die meisten internationalen Organisationen sind bislang vor internationalen Gerichten nicht vorgesehen ${ }^{66}$ und nationale Gerichte lassen Klagen, auch aufgrund der meist weitreichenden Immunität internationaler Organisationen, nicht zu. ${ }^{67}$ In der Praxis kommt der Bindung an die allgemeinen Rechtsgrundsätze (hard law) bislang deshalb nicht mehr Bedeutung als der Selbstbindung (soft law) internationaler Organisationen an Menschenrechtsstandards zu, die sich einige internationale Organisationen in menschenrechtlich besonders relevanten Bereichen auferlegt haben. ${ }^{68}$ Zudem bestehen Abgrenzungsschwierigkeiten zum Gewohnheitsrecht und methodische Unsicherheiten bei der Feststellung allgemeiner Rechtsgrundsätze ${ }^{69}$ weshalb die so begründete Bindung an menschenrechtliche Standards für die aus ihnen Verpflichteten schwer erkennbar ist.

Bei der Bindung „Privater“ an menschenrechtliche Standards ist man insoweit weiter, als diese zumindest bei schwerwiegenden Völkerrechtsverletzungen (Kriegsverbrechen), die alle auch die Verletzung menschenrechtlicher Standards darstellen, persönlich vor Gericht zur Verantwortung gezogen werden können.

Jedoch besteht bislang keine umfassende direkt Bindung „Privater“ an Menschenrechtsstandards. Bei bestehenden Initiativen zu Selbstregulierung von Firmen, die oft inhaltlich nur auf einzelne Themen des Menschenrechtsschutzes beschränkt sind, ${ }^{70}$ ist als großes Problem die Durchsetzbarkeit dieser zu sehen, da, wenn überhaupt eine Überwachung der codes of conduct vorgesehen ist, diese in der Regel nur firmenintern erfolgt. ${ }^{71}$ Auch das innerstaatlichen Deliktsrecht führt, wenn überhaupt, nur in Einzelfällen dazu, dass „Private“ für die Verletzung menschenrechtlicher Inhalte zur Verantwortung gezogen werden können. ${ }^{72}$

Eine umfassende Bindung an menschenrechtliche Standards, die auch vor Gerichten geltend gemacht werden kann, besteht folglich weiterhin nur für Staaten.

64 s. ausführliche Analyse der Gründungsverträge von VN, IBRD, IDA und IWF, Janik, (Fn. 1), 331 ff.; s. bezogen auf die VN, $336 \mathrm{f}$.

65 s. Fn. 49.

66 Janik, (Fn. 1), 546.

67 s. bezogen auf die VN Peters, (Fn. 4), 30 und 44.

68 s. ausführlich zur Selbstbindung der VN, der IBRD und des IWF, Janik, (Fn. 1), 203 ff.

69 Moos, (Fn. 18), 297.

70 Hennings, (Fn. 55), 61; Gründe für die Selbstregulierung von Firmen sind: Angst, dass ansonsten Regierungen auf nationaler oder internationaler Ebene eingreifen, die Besorgnis um den eigenen guten Ruf, der Wettbewerb mit anderen Firmen oder bereits gemachte positive Erfahrungen, s. Haufler, A Public Role for The Privat Sector- Industry Self-Regulation in a Global Economy, 2001, $105 \mathrm{f}$.

71 Hennings (Fn. 55), 63.

72 Lehnardt, (Rn. 26), 252. 


\section{Zurechnung menschenrechtsverletzender Handlungen zu nichtstaatlichen Akteuren}

Die Zurechnung bezieht sich auf das Innenverhältnis zwischen Völkerrechtssubjekt und dem im konkreten Fall handelnden Akteur. ${ }^{73}$ Sie gibt Auskunft darüber, ob die Handlung eines Handlungsagenten als Handeln des Völkerrechtssubjekts zu qualifizieren ist. ${ }^{74}$ Die bestehende Vielzahl an Zurechnungssubjekten, sowie die Frage nach den anwendbaren Zurechnungsregeln bereiten Probleme.

\section{Zurechnung menschenrechtsverletzender Handlungen zu internationalen Organi- sationen}

Die Draft Articles on the Responsibility of International Organizations (DARIO), eine Zusammenfassung existierender Regeln über die Haftung internationaler Organisationen $\mathrm{zu}$ einem integrativen Haftungssystem durch die International Law Commission (ILC), ${ }^{75}$ stellen spezielle Zurechnungsregeln für das Handeln internationaler Organisationen auf. Kapitel II der DARIO beschäftigt sich damit, wann ein Handeln einer internationalen Organisation zurechenbar ist (Art. 6 DARIO (Conduct of organs or agents of an international organization); Art. 7 DARIO (Conduct of organs of a State or organs or agents of an international organization placed at the disposal of another international organization); Art. 8 DARIO (Excess of authority or contravention of instructions); Art. 9 DARIO (Conduct acknowledged and adopted by an international organization as its own)). Kapitel IV DARIO regelt außerdem die abgeleitete, sekundäre Verantwortlichkeit internationaler Organisationen für durch Staaten oder durch andere internationaler Organisationen begangene Verletzungen des Völkerrechts. ${ }^{76}$ Es fehlt bislang an einer umfassenden gewohnheitsrechtlichen Anerkennung der DARIO. ${ }^{77}$ Gewohnheitsrechtlich anerkannt ist nur, dass auch internationale Organisationen völkerrechtlich verantwortlich sein können, soweit sie eigene Rechtssubjektivität besitzen und das betreffende Verhalten der Organisation zurechenbar ist (vgl. Art. 3 DARIO). ${ }^{78}$ Vielfach wird zudem angenommen, dass das Kriterium der effektiven Kontrolle über die Zuweisung des Verhaltens entscheidet (vgl. Art. 7 DARIO), völkergewohnheitsrechtlich anerkannt ist. ${ }^{79}$ Dass letztgenannte Regel kein gesetztes Recht ist, zeigt der Vorschlag des

73 s. bezogen auf Staaten, Moos, (Fn. 18), 81.

74 Ibid.

75 Ruffert/Walter, (Fn. 14),§ 7 Rn. 235.

76 Von "sekundärer abgeleiteten Verantwortlichkeit" spricht Janik, (Fn. 1), 37.

77 ILC, Report on the work of its fifty-fourth session 2002, UN Doc.Suppl.No. 10 (A/57 (10), Rn. 473; Larsen, Kjetil Mujezinović, Attribution of Conduct in Peace Operations; The "Ultimate Authority and Control" Test, EJIL, 2008, 509, 518.

78 Thallinger, Grundrechte und extraterritoriale Hoheitsakte, Auslandseinsätze des Bundesheeres und EMRK, 2008, 29; Hofmann, (Fn. 19), 26; vgl. auch ILC, Report of its fifty-third session, 23.4.2001-1.6.2001 sowie 2.7.2001-1.8.2001, UN Doc. A/56/10, Art. 57, Rn. 2, 141; Moos, (Fn. 18), 95.

79 vgl. Gaja, Second Report on Responsibility of International Organizations vom 2.4.2004, UN Doc. A/CN.4/541, Rn. 40; Für Krieger gebietet dies die Rechtslogik, auch wenn die Regel noch kein Völkergewohnheitsrecht darstelle, Krieger, Die Verantwortlichkeit Deutschlands nach der EMKR für seine Streitkräfte im Auslandseinsatz, ZaöRV, 2002, 669, 677 f.; Moos, (Fn. 18), 95; Ruffert/Walter, (Fn. 14), § 7, Rn. 222. 
CDDH bezüglich der Zurechnung von Handlungen zur EU oder ihren Mitgliedstaaten nach dem geplanten Beitritt der EU zur EMRK. ${ }^{80}$ Vorgesehen ist, dass:

"3. Accession to the Convention and the protocols thereto shall impose on the European Union obligations with regard only to acts, measures or omissions of its institutions, bodies, offices or agencies, or of persons acting on their behalf. Nothing in the Convention or the protocols thereto shall require the European Union to perform an act or adopt a measure for which it has no competence under European Union law.

4. For the purposes of the Convention, of the protocols thereto and of this Agreement, an act, measure or omission of organs of a member State of the European Union or of persons acting on its behalf shall be attributed to that State, even if such act, measure or omission occurs when the State implements the law of the European Union, including decisions taken under the Treaty on European Union and under the Treaty on the Functioning of the European Union. This shall not preclude the European Union from being responsible as a co-respondent for a violation resulting from such an act, measure or omission, in accordance with Article 36, paragraph 4, of the Convention and Article 3 of this Agreement." 81

Der vorgeschlagene rein formale Ansatz der Zurechnung von Handlungen zur EU oder den Mitgliedstaaten (Rechtsträgerlösung) weicht von der in Art. 7 DARIO geregelten Organleihe ab, die vorsieht, dass das Verhalten des handelnden staatlichen Organs der internationalen Organisation, in dessen Dienst es steht, zugerechnet wird, wenn diese die effektive Kontrolle über das fragliche Verhalten ausübt.

Neben den DARIO besteht das Recht der Staatenverantwortlichkeit, das durch die ILC in ihren Draft Articles on Responsibility of States for Internationally Wrongful Acts (ASR) kodifiziert wurde. ${ }^{82}$ Die ASR sind zwar nicht vertraglich bindend, aber weitestgehend gewohnheitsrechtlich anerkannt. ${ }^{83}$ Betrachtet man das Verhältnis der Regeln der ASR zu denen der DARIO, sind letztere als leges specialis zu erachten, wenn eine internationale Organisation beteiligt ist und als Zurechnungssubjekt entweder die internationale Organisation oder der Mitgliedstaat in Betracht kommt. ${ }^{84}$ Hingegen ist das Recht der ASR heranzuziehen, wenn die Frage zu beantwortet ist, ob eine bestimmte Handlung überhaupt einem Staat zurechenbar ist. ${ }^{85}$ Diese Abgrenzungsregeln finden gegenwärtig jedoch kaum Beachtung. Sowohl in der Rechtsprechung, als auch im Schrifttum überwiegt weiterhin die Zurechnung nach den Regelungen des Rechts der ASR, die gegebenenfalls auch mutatis mutandis auf internationale Organisation angewendet werden. ${ }^{86}$

80 Gem.Art. 64 DARIO finden die DARIO keine Anwendung, wenn speziellere Regeln greifen, weshalb Menschenrechtsverträge eigene abweichende Regeln enthalten können; s. bezogen auf die ASR Künzli, Zwischen Rigidität und Flexibilität: Der Verpflichtungsgrad internationaler Menschenrechte, 2001, 94; vgl. auch Hofmann (Fn. 19), 33, der sich dafür ausspricht, dass die DARIO auf die EU keine Anwendung finden sollten.

81 Final Report of the CDDH, (Fn. 35), Art. 1 Rn. 3 f., 5.

82 ICL, Responsibility of States for Internationally Wrongfull Acts, 2001, von der International Law Commission in ihrer 53. Sitzung 2001 angenommen.

83 s. z.B. Zwanenburg, Accountability of peace support operations, 2005, 51.

84 Moos, (Fn. 18), 97; ILC. Report of its sixty-first session, 4.5.2009-5.6.2009 sowie 6.-7.8.2009, UN Doc. A/64/10, Artikel 6, Rn. 1, 62.

85 Moos, (Fn. 18), 97; ILC. Report of its sixty-first session, (Fn. 84), Artikel 6, Rn. 4, 65.

86 vgl. z.B. Zwanenburg, (Fn. 83), 69 ff., 126 f., 209 ff., 221; Moos, (Fn. 18), 96. 


\section{Exklusiv-oder Parallelhaftung von internationalen Organisationen und ihren Mit- gliedstaaten}

Die Rechtsprechung des EGMR in der Rechtssache Behrami/Seramati verdeutlicht, dass der gegenwärtige Zustand, in dem zwar Zurechnungsregeln bestehen, die es ermöglichen, zu bestimmen, ob Zurechnungssubjekt einer menschenrechtsverletzenden Handlungen entweder ein Mitgliedstaat einer internationalen Organisation oder die internationale Organisation selbst ist, letztgenannte aber nicht an den menschenrechtlichen Vertrag gebunden ist, es schwer machen, die Zurechnungsfrage zu beantworten. Der EGMR, der die DARIO anwendete, hatte nur die Frage nach der Verantwortung der Konventionsstaaten und nicht die der internationalen Organisation als Nichtvertragsparteien zu beantworten. ${ }^{87}$ Nach dem Behrami-Ansatz führte die Zurechnung zu einer internationalen Organisation, in der Art eines Nullsummenspiels, dazu, dass eine konkrete Maßnahme dann nicht dem Konventionsstaat zugerechnet wird. ${ }^{88}$ Sollte deshalb dafür plädiert werden, dass wenn nur ein Staat, aber nicht die internationale Organisation, in deren Rahmen er handelt, Partei eines völkerrechtlichen (menschenrechtsschützenden) Vertrages ist, die Regeln der ASR angewendet werden sollten? ${ }^{89}$ Trotz Beteiligung einer internationalen Organisation ist für das entscheidende Gericht letztendlich in dieser Konstellation nämlich nur relevant, ob die Verletzungshandlung dem Konventionsstaat zurechenbar ist. Es handelt sich strenggenommen nicht um eine Situation, in der als Zurechnungssubjekt entweder die internationale Organisation oder der Mitgliedstaat in Betracht kommt. Inwieweit dies nur ein formalistischer Gesichtspunkt ist, hängt davon ab, ob die Regeln der ASR und die Regeln der DARIO zum selben Ergebnis der Zurechnung gelangen. ${ }^{90}$ Relevant wird insoweit, ob das in Art. 8 ASR und Art. 7 DARIO genannte Kriterium der effektiven Kontrolle gleich ausgelegt wird. ${ }^{91}$ Aus Gründen der Rechtssicherheit wäre es zu begrüßen, wenn die Anwendung beider Regime zu demselben Ergebnis führen würde..$^{2}$ Gegenwärtig ist der Inhalt dieses Kriteriums in beiden Haftungsregimen jedoch mit großer Unsicherheit behaftet.

Weniger Brisanz hätte die Rechtsprechung des EGMR in der Rechtssache Behrami/ Seramati besessen, hätte der EGMR eine parallele Verantwortlichkeit der Mitgliedstaaten angenommen ${ }^{93}$ Für eine simultane Zurechnung zu beiden Völkerrechtssubjekten lassen sich Abgrenzungsprobleme anführen. Es ist z.B. durchaus möglich, dass die effektive Kontrolle über das Handeln von Soldaten bei einer von einer internationalen

87 Peters, (Fn. 4), 35.

88 Ibid.

89 Vorschlag von Peters, Ibid.

90 Ibid.

91 Ibid., mit Nennung des Art. 5 ILC-Entwurfs zu internationalen Organisationen, der dem heutigen Art. 7 DARIO entspricht.

92 Ibid.; Peters erklärt, dass die Unterschiede in der Funktion der Rechtsregime (ASR: Beantwortung der Frage, ob ein Verhalten überhaupt einem Hoheitsträger zurechenbar ist; DARIO: Aufteilung der Verantwortung zwischen Staaten und internationalen Organisationen) keine abweichende Interpretation des identischen Begriffs rechtfertigen könne.

93 Für eine simultane Zurechnung s. z.B. Moos, (Fn. 18), 108 f.; a. A. Wilde, Enhancing Accountability at the International Level: The Tension between International Organizations and Member State Responsibility and the Underlying Issues at Stake Advancing the Effectiveness of International Law: Is U.N. Reform Necessary, in: 12 ILSA JICL, 2005/2006, 395, $411 \mathrm{f}$. 
Organisation geführten Operation weder ausschließlich durch die internationale Organisation, noch ausschließlich durch den Entsendestaat ausgeübt wird ${ }^{94}$ Kritiker einer parallelen Zurechnung bemängeln, dass das Interesse des Opfers zu Lasten der Funktionsfähigkeit der Organisation überbewertet werde. ${ }^{95}$ Die weiterbestehende volle Verantwortlichkeit der Mitgliedstaaten für Handeln der internationalen Organisation könne nämlich zur Folge haben, dass die Mitgliedstaaten dauernd intervenieren und somit die Aufgabenerfüllung der Organe der internationalen Organisation behindern könnten. ${ }^{96}$ Ferner wird befürchtet, dass sich dies negativ auf die Bereitschaft der Mitgliedstaaten zum Streit- und Personaleinsatz auswirken könnte. ${ }^{97}$

Eine Ideallösung existiert nicht: entweder man entscheidet sich dafür, die Mitgliedstaaten nicht für all ihr Handeln im Rahmen von internationalen Organisationen verantwortlich zu machen und trägt somit der realen Gegebenheit Rechnung, dass internationale Organisationen eine Handlungseinheit darstellen, die (graduell) autonom von ihren Mitgliedstaaten ist. ${ }^{98}$ Dies bedeutet jedoch gegenwärtig auch die Akzeptanz von Rechtsschutzlücken (siehe die Entscheidung des EGMR in der Rechtssache Behrami/ Seramati), außer die internationale Organisation selber verfügt über ein eigenes menschenrechtliches Rechtsschutzsystem. ${ }^{99}$ Oder man befürwortet grundsätzlich eine doppelte Verantwortlichkeit von Mitgliedstaaten und internationaler Organisation, wodurch Rechtsschutzlücken vermieden werden können, jedoch die realen Gegebenheiten nicht immer abgebildet werden. Eine Lösung dieses Dilemmas verspricht nur eine umfassende Bindung internationaler Organisationen an menschenrechtliche Standards und der Aufbau eines effektiven Rechtsschutzes gegen internationale Organisationen. ${ }^{100}$

Geschieht dies, wie durch den geplanten Beitritt der EU zur EMRK, besteht jedoch weiterhin das Problem, dass eine Abgrenzung der Verantwortlichkeit nicht immer möglich ist, bzw. schwer fällt. Diskutiert wurde bezüglich des geplanten Beitritts der EU zur EMRK insoweit die Schwerpunktlösung, die vorsieht, dass die EU und ihre Mitgliedstaaten intern bestimmen, wer sich gegenüber den Konventionsorganen verantworten muss, ${ }^{101}$ die Rechtsträgerlösung, die als zutreffenden Beschwerdegegner immer diejenige Vertragspartei ansieht, deren Behörde oder dessen sonstigem Verwaltungsträger die mit der Beschwerde angegriffene Maßnahme zuzurechnen ist, ${ }^{102}$ und die Einführung des Mechanismus des Mitbeklagten (co-respondent). ${ }^{103}$ Als Mitbeklagter nimmt die in-

94 Moos, (Fn. 18), 109.

95 Hirsch, The Responsibility of International Organization Towards Third Parties: Some Basic Principles, 1995, 155.

96 Wilde, (Fn. 93), 404 f.; Zwanenburg, (Fn. 83), 127; EGRM, Appl.nos.71412/01 und 78166/01, Behrami and Saramati v. France u.a., Entscheidung v. 2.5.2007, Rn. 149.

97 Krieger, (Fn. 79), 696 ff.; Zwanenburg, (Fn. 83), 127.

98 Peters, (Fn. 4), 21.

99 Die trifft bislang wohl nur auf die EU zu. Wobei trotzdem der Bedarf gesehen wird, diese an einen externen Menschenrechtskatalog (EMRK) zu binden.

100 Moos, (Fn. 18), 117.

101 Gutachtenbericht, EuGH, Gutachten vom 28.3.1996, G 2/94, Beitritt zur EMRK, Slg.1996, I-1759, I.6.

102 Winkler, Der Beitritt der EG zur EMRK, 2000, 54.

103 3rd working meeting of the CDDH informal working group in the accession of the EU to the ECHR(CDD-UE) with the European Commission, 19.-22.10.2010, CDDH$\mathrm{UE}(2010) 12$, C. 3. 
ternationale Organisation neben dem eigentlich beklagten Mitgliedstaaten als gleichwertiger Verteidiger am Verfahren teil und umgekehrt, die Mitgliedstaaten, wenn sie den Status des Mitbeklagten innehaben, auch an Verfahren, in denen die internationale Organisation die eigentliche Beklagte ist. ${ }^{104}$ Als gleichwertiger Verfahrensbeteiligter folgt auch die Pflicht zur Urteilsumsetzung. ${ }^{105}$ Der vorliegende Vorschlag der CDDH kombiniert die Rechtsträgerlösung mit der eines nicht verpflichtenden Mechanismus des Mitbeklagten.

\section{Zurechnung menschenrechtsverletzender Handlungen zu "Privaten"}

Das Völkerrecht unterscheidet zwischen privatem und staatlichem Verhalten. ${ }^{106}$ Das Verhalten „Privater“ als solches zeitigt grundsätzlich keine völkerrechtliche Verantwortlichkeit. ${ }^{107}$ Jedoch bestehen oben beschriebene Ausnahmen, insbesondere die der völkerstrafrechtlichen Verantwortlichkeit von „Privaten“. Problem im Völkerstrafrecht ist meist die Beteiligung vieler auf unterschiedlichen hierarchischen Ebenen. ${ }^{108}$ Die militärische oder zivile Kommandostruktur bei der Begehung der Tat stellt oft den entscheidenden Faktor dar. ${ }^{109}$ Es handelt sich um eine anspruchsvolle Aufgabe, befriedigende Antworten auf die Frage, wie Einzelpersonen für das aus kollektiven Aktivitäten resultierende Unrecht mittels Zurechnung verantwortlich gemacht werden können, zu finden. ${ }^{110}$ Eine Reihe von Fragestellungen, wie Beteiligungsformen, Vorgesetztenverantwortlichkeit, Vorsatzerfordernisse und Handeln auf Befehl werden im Römischen Statut (in Part III: “General Principles of Criminal Law" (Art. 22-33 IStGHSt)) zwar angesprochen, eine dogmatisch einwandfreie Lösung wird bislang jedoch nicht geliefert. ${ }^{111}$ Die völkerstrafrechtliche Zurechnungslehre ist insgesamt bislang unterentwickelt, wenn man überhaupt schon von einer eigenen Lehre sprechen kann. ${ }^{112}$ Die Herausforderung besteht darin eine funktional leistungsfähige völkerstrafrechtliche Zurechnungslehre zu entwickeln, ${ }^{113}$ die die breite Akzeptanz der Nationalstaaten fin-

104 Ibid.

105 Ibid., C.3.I.

106 Lehnardt, (Fn. 26), 82.

107 Tomuschat, International law: ensuring the survival of mankind on the eve of a new century, RdC 182 (1999), 274.

108 Safferling, (Fn. 51), § 5 Rn. 2,.

109 Ibid.

110 Safferling/Kirsch, Völkerstrafrechtspolitik, in: dies. (Hrsg.), Völkerstrafrechtspolitik, 2014, $1,5$.

111 Safferling, (Fn. 51), § 5 Rn. 2.

112 Ibid.,§ 5 Rn. 1, der versucht eine solche zu entwickeln; Werle hebt hingegen die Fortschritte der Dogmatisierung des Völkerstrafrechts in den letzten Jahren hervor, s. Werle, Die Zukunft des Völkerstrafrechts, in: Grundmann et al. (Hrsg.), FS 200 Jahre Juristische Fakultät der Humboldt-Universität zu Berlin, 2010, 1219, 1231.

113 Ambos, Internationales Strafrecht, Strafanwendungsrecht-Völkerstrafrecht- europäisches Strafrecht, 2. Aufl., 2008, § 7 Rn. 1. 
det, ${ }^{114}$ obwohl diese durch national bestehende unterschiedliche Zurechnungsmodelle geprägt sind. ${ }^{115}$

\section{Zurechnung einer durch „Private“ begangenen menschenrechtsverletzenden Hand- lung zum Staat}

Grundsätzlich sind Staaten völkerrechtlich nicht für das Verhalten „Privater“ verantwortlich. ${ }^{16}$ Sie können nur für ihr eigenes Verhalten verantwortlich gemacht werden. ${ }^{117}$ Jedoch werden Staaten erst durch natürliche Personen handlungsfähig. ${ }^{118}$ Primär handeln sie durch ihre Organe, deren Verhalten ihnen nach den Regeln der Staatenverantwortlichkeit zurechenbar ist (Art. 4 ARS, de iure-, und de facto-Organe), ${ }^{119}$ unabhängig davon, ob die konkrete Handlung vom Staat gewollt oder kontrolliert wurde und diese eine Ausübung von hoheitlichen Funktionen darstellt. ${ }^{120}$ De iure-Organe im völkerrechtlichen Sinn sind alle Personen, die als Funktionsträger innerhalb des Staatsapparats formell mit der Wahrnehmung exekutiver, legislativer und judikativer Aufgaben betraut worden sind. ${ }^{121}$ Bei Bestehen von vollständiger Abhängigkeit (complete dependence) einer Person, Gruppe oder Einheit vom Staat, ${ }^{122}$ kann er sich seiner Verantwortung nicht dadurch entziehen, dass er leugnet, dass diese de facto-Organe in seiner Rechtsordnung den Organstatus innehaben. ${ }^{123}$

Bei fehlender Organstellung muss eine besondere Beziehung zwischen dem privaten und dem staatlichen Verhalten festgestellt werden, welche die Zurechnung rechtfertigt. ${ }^{124} \mathrm{Im}$ Ergebnis muss das Verhalten als staatliches Verhalten erscheinen. ${ }^{125}$ Es besteht die Möglichkeit der funktionsbegründeten (Art. 5 und 9 ARS) und der steuerungsbegründeten Zurechnung des Verhaltens „Privater“ zum Staat (Art. 8 ARS). ${ }^{126}$ Ferner

114 Burghardt, Die Rechtsvergleichung in der völkerstrafrechtlichen Rechtsprechung, in: Beck et al. (Hrsg.), Strafrechtsvergleichung als Problem und Lösung, 2010, 235.

115 Safferling, (Fn. 51), § 5 Rn. 1.

116 Lehnardt, (Fn. 26), 82.

117 Ibid.

118 Wilms, Staatsrecht, Band 1, Staatsorganisationsrecht unter Berücksichtigung der Föderalismusreform, 2007, Rn. 378.

119 s. Schröder, Verantwortlichkeit, Völkerstrafrecht, Streitbeilegung und Sanktionen, in: Graf Vitzthum (Hrsg.), Völkerrecht, 579, 593.

120 IGH, Case concerning the application of the Convention on the Prevention and Punishment of the Crime of Genocide (Bosnia and Hercegovina v. Serbia and Montenegro), Urteil v. 26.2.2007, Rn. 393 .

121 s. Crawford, First report on state responsibility, Addendum 5, Rn. $166 \mathrm{f}$.

122 IGH, Case concerning the application of the Convention on the Prevention and Punishment of the Crime of Genocide (Fn. 120), Rn. 392; Military and Paramilitary Activities in and against Nicaragua (Nicaragua v. United States of America), Merits, ICJ Report 1986, 14, 62-63, Rn. $109 \mathrm{f}$.

$123 \mathrm{Ob}$ man das Handeln dieser Personen durch eine weite Auslegung des Begriffs de iureOrgans oder die Einführung der Extrakategorie des de facto-Organs umfassend dem Staat zurechnet, ist letztendlich praktisch ohne Bedeutung. Commentary to Art. 4, para 11.

124 s. hierzu ausführlich: Lehnardt, (Fn. 26), 86.

125 Schröder, (Fn. 119), 589.

126 Seibert-Fohr, Die völkerrechtliche Verantwortung des Staats für das Handeln von Privaten: Bedarf nach Neuorientierung? ZaöRV 2013, 37, 42. 
kann ein Staat das Verhalten „Privater“ als eigenes anerkennen und annehmen (Art. 11 ARS). ${ }^{127}$ Probleme bereitet, dass es auf völkerrechtlicher Ebene bislang an einer Definition von hoheitlicher Gewalt fehlt, ${ }^{128}$ dem zentralen Element der funktionsbegründeten Zurechnung gemäß Art. 5 oder 9 ASR. ${ }^{129}$ Die ILC nimmt an, dass es sich bei hoheitlicher Gewalt um die Übertragung der Ausübung von Aufgaben handelt, die normalerweise von den Organen des Staates ausgeübt werden. ${ }^{130}$ Die Ausweitung der Übertragung von „Hoheitsgewalt“ auf Private und internationale Organisationen macht es jedoch immer schwerer zu bestimmen, welche Aufgaben normalerweise von den Organen eines Staates ausgeübt werden. Erhebliche Unsicherheiten bestehen auch bezüglich der durch Art. 8 ASR vorgesehenen steuerungsbegründeten Zurechnung des Verhalten „Privater“ zum Staat, da dieser nicht vorgibt welche Art von Kontrolle des Verhaltens gefordert wird. Während der IGH im Genozid-Fall für die Zurechnung des Verhaltens eine effektive Kontrolle (effective control) über die spezifische Verletzungshandlung verlangte, ${ }^{131}$ forderte die Berufungskammer des Internationalen Strafgerichtshofs für das frühere Jugoslawien nur eine generelle Kontrolle (overall control) über das Tätigkeitsfeld der handelnden Personen. ${ }^{132}$ Probleme bereiten hierbei nicht nur die Verwendung verschiedener Begrifflichkeiten, sondern auch die fehlende Erläuterung des Inhalts dieser Begriffe. ${ }^{133}$

Die Frage, ob zudem durch Beihilfehandlungen zu durch „Private“ begangene Völkerrechtsverletzungen die Verantwortlichkeit eines Staates ausgelöst werden kann, ${ }^{134}$ stellt sich überhaupt nur in Ausnahmefällen, da die Mehrheit der Normen des Völkerrechts weiterhin nur Staaten adressiert ${ }^{135}$ und nicht, wie hierfür erforderlich, sowohl den Staat als auch Privatpersonen. ${ }^{136}$ Zudem stellen diese Regelungen, die auch keinen Eingang in die Regeln der ASR gefunden haben, kein Völkergewohnheitsrecht dar. ${ }^{137}$

Eine Zurechnung zum Staat erfolgt unstrittig nicht, wenn „Private“ losgelöst vom staatlichen Kontext handeln und keinerlei Verbindung zu staatlichen Funktionsträgern

127 Ibid.

128 Vgl. ICSID, Noble Ventures, Inc.v. Romania, ARB/01/11, Entscheidung v. 12.10.2005, Rn. 82; Lehnardt, (Fn. 26), 157.

129 Lehnardt, (Fn. 26), 156.

130 Commentary to draft Art. 7, para 18: Report of the ILC, 26th Session, ILC Yearbook 1974, Vol II (1), 282.

131 IGH, Case Concerning the Application of the Convention on the Prevention and Punishment of the Crime of Genocide, (Fn. 120), Rn. $388 \mathrm{ff}$.

132 Internationale Strafgerichtshof für das frühere Jugoslawien, Prosecutor v. Dusko Tadić, Judgment, Appeals Chamber, Case No.IT-94-1-A, 15.6.1999, Rn. 145, 156.

133 Lehnardt, (Fn. 26), 127.

134 MCCorquodale/Simons, Responsibility Beyond Borders: State Responsibility for Extraterritorial Violations by Corporations of International Human Rights Law, MLR 70, 2007, 598, $613 \mathrm{ff}$.

135 de Frouville, Attribution of Conduct to the State: Privat Individuals, in: Crawford (Fn. 53), 277; Der IGH akzeptierte, dass die Fedaral Republic of Yugoslavia sich der Beihilfe zur Straftat des Genozides, durchgeführt durch den nichtstaatlichen Akteur der Republik Sprska in Srebrenica, schuldig gemacht haben könnte, auch wenn er sie im Ergebnis ablehnte; Case Concerning the Application of the Convention on the Prevention and Punishment of the Crime of Genocide, (Fn. 120), Rn. $416 \mathrm{ff}$.

136 de Frouville, (Fn. 135), 277.

137 Ibid. 
haben. ${ }^{138}$ Wenn das Verhalten „Privater“ dem Staat nicht zurechenbar ist, kann dieses gleichwohl seine völkerrechtliche Verantwortlichkeit auslösen. Völkerrechtliche Schutzpflichten im Bereich der Menschenrechte und des humanitären Völkerrechts können den Staat zur Ergreifung von Maßnahmen verpflichten, die auf Kontrolle und Überwachung, sowie Verbot und Sanktionierung von privaten Rechtsverletzungen gerichtet sind. ${ }^{139}$ Im Schrifttum ist deshalb die Ansicht verbreitet, dass es keiner Änderung des völkerrechtlichen Haftungsregimes bedarf, um den Gefährdungen durch „Private“ begegnen zu können. ${ }^{140}$ Die völkerrechtlichen Schutzpflichten stellten eine adäquate Lösung dar, die Schutzlücken vermeide. ${ }^{141}$ Bislang fehlt es jedoch an Systematik, einer einheitlichen Anwendung von Rechtsgrundsätzen, der Klärung ihrer Voraussetzungen und ihrer Rechtsfolgen. ${ }^{142} \mathrm{Zu}$ begrüßen sind deshalb Versuche, eine Sorgfaltspflichtsdogmatik für das universelle Völkerrecht zu entwickeln, ${ }^{143}$ die Regeln aufstellt, mit denen es möglich ist, noch nicht behandelte und neue Fälle nach einheitlichem Muster und trotzdem einzelfallgerecht lösen zu können. ${ }^{144}$

\section{Die Zurechnung privaten Handelns zu internationalen Organisationen}

Internationale Organisationen handeln, wie Staaten, in erster Linie durch ihre Organe. Sie äußern ihren Willen und erfüllen ihre Pflichten durch diese. ${ }^{145} \mathrm{Ihr}$ Verhalten ist der internationalen Organisation zurechenbar (Art. 6 DARIO). ${ }^{146}$ Zudem ist ihnen das Verhalten ihrer Agenten, wenn diese Aufgaben der Organisation, die sich nach deren internen Regeln bestimmen, erfüllen, zurechenbar (Art. 6 DARIO). Ein Agent ist laut IGH jede Person, die durch eine internationale Organisation damit beauftragt wurde, eine ihrer Aufgaben auszuführen oder ihr bei der Aufgabenerfüllung zu helfen, unabhängig davon, ob sie von dieser offiziell bezahlt wird oder ob sie langfristig angestellt wurde. ${ }^{147}$ Meist besteht sowohl bei Organen als auch bei Agenten eine formale Verbindung zur internationalen Organisation dergestalt, dass ihre Rolle und ihr Platz in der Struktur der internationalen Organisation in den Gründungverträgen oder durch Sekundärrecht geregelt werden. ${ }^{148}$ Ansonsten ist aufgrund der Bestimmungen der jeweiligen interna-

138 Seibert-Fohr, (Fn. 126), 42; ILC, ARS Commentaries (Anm. 11), Chapter II, para.4.

139 Lehnardt, (Fn. 26), 225; de Frouville, (Fn. 135), 277.

140 Seibert-Fohr, (Fn. 126), 60.

141 Ibid.

142 Stahl, Schutzpflichten im Völkerrecht-Ansatz einer Dogmatik, Ein Beitrag zu Grund, Inhalt und Grenzen der völkerrechtlichen Schutzpflichtendogmatik im Bereich konventionell geschützter Menschenrecht, 2012, 24.

143 Seibert-Fohr, (Fn. 126), 49 ff.; siehe ausführlich Stahl, (Fn. 142).

144 Stahl, (Fn. 142), 426.

145 Klein, The Attribution of Acts to International Organizations, in: Crawford et al. (Fn. 53), 297, 298.

146 Schweisfurth, Völkerrecht, 2006, 235.

147 Reparation for Injuries Suffered in the Service of the United Nations, ICJ Report 1929, 174, 177; Gaja, (Fn. 79), Rn. 17.

148 Klein, (Fn. 145), 298. 
tionalen Organisation festzustellen, welche Funktionen diese innehat und ob diese Funktionen von privatem Personal ausgeübt wird. ${ }^{149}$

Die DARIO enthalten keine Art. 8 ASR entsprechende Regelung, die vorsehen würde, dass von einer internationalen Organisation geleitetes oder kontrolliertes Verhalten privater Personen oder Personengruppen der internationalen Organisation zurechenbar ist. Kein Problem stellt dies dar, wenn das Verhalten, z.B. privaten Militärpersonals nach den Regeln der Staatenverantwortlichkeit dem Staat zurechenbar ist und sodann anhand der DARIO nur geklärt werden muss, ob die Verletzungshandlung dem Staat selbst oder der internationalen Organisation zurechenbar ist. Gemäß Art. 7 DARIO ist für diese Abgrenzung auf das Vorliegen von effektiver Kontrolle (effective control) über das Verhalten abzustellen. ${ }^{150}$

Es ist aber auch durchaus möglich, dass das Verhalten „Privater“ keinem Staat zurechenbar ist. Beispielsweise bedienen sich internationale Organisationen auch selbst privater Militärfirmen, zu nennen ist etwa deren Einsatz im Rahmen von PeacekeepingMissionen der Vereinten Nationen. ${ }^{151}$ Möglich wäre zum einen, dass in einer solchen Situation „Private“ als Agenten der internationalen Organisation betrachtet werden können, zum anderen bestünde die Möglichkeit auch hier das Kriterium der effektiven Kontrolle über die Zuweisung des Verhaltens als entscheidend zu betrachten, das viele für gewohnheitsrechtlich anerkannt erachten. Es darf im Ergebnis keinen Unterschied für die Zurechnung des Verhaltens „Privater“ zu einem Rechtssubjekt machen, ob es sich um eine internationale Organisation oder einen Staat handelt, der das Verhalten des „Privaten“ kontrolliert.

\section{Zwischenfazit}

Bislang fehlt es an Konsistenz und Kohärenz bezüglich der bei Beteiligung von internationalen Organisationen anwendbaren Zurechnungsregeln. In der Praxis bereitet die Abgrenzung der Anwendbarkeit der DARIO und der ASR Probleme, wobei es im Ergebnis keinen Unterschied für die Zurechnung eines Verhaltens zu einem Staat machen darf, ob die Regeln der ASR oder die der DARIO angewendet werden. Die beiden Haftungsregime sind dahingehend im Gleichklang auszulegen.

Wenn ein Verhalten nach den DARIO einer internationalen Organisation zurechenbar ist, hat diese Zurechnung unter (fast) keinem bestehenden Menschenrechtsregime bislang zur Folge, dass diese auch selbst für Menschenrechtsverletzungen zur Verantwortung gezogen werden könnte. Die Rechtsprechung in der Rechtssache Behrami/Seramati hat verdeutlicht, dass Rechtsschutzlücken die Folge seien können. Mit der Aner-

149 Gaja, (Fn. 79), Rn. 17; Ruffert/ Walter, (Fn. 14), § 7, Rn. 221.

150 Klein, (Fn. 145), 299.

151 Zum Einsatz privater Militärfirmen durch die EU, s. z. B. White./MacLeod, EU Operations and Private Military Contractors: Issus of Corporate and Institutional Responsibility, EJIL 19 (2008), 965 ff.; durch die NATO, s. z.B. Bayles./Holmqvist, The increasing role of private military and security companies. Study requested by the European Parliament's Subcommittee on Security and Defence, 2007, 18; durch die UN s. z. B. Note of the Under SecretaryGeneral for Management, Summary of the work of the Fifth Committee during the first part of the resumed 56th session, 4.-19.3.2002. 
kennung internationaler Organisationen als neue Zurechnungssubjekte für Verletzungen völkerrechtlicher Normen ist, bezogen auf die Menschenrechte, nicht zugleich die Anerkennung als Verantwortungssubjekte für Verletzungen der Menschenrechte einhergegangen. Es ist deshalb die Parallelzurechnung von Verhalten zur internationalen Organisation und ihren Mitgliedstaaten zu befürworten, zumindest bis internationale Organisationen für die Verletzungen menschenrechtlicher Inhalte auch selbst zur Verantwortung gezogen werden können.

Das Verhalten „Privater“ als solches zeitigt nur in Ausnahmefällen völkerrechtliche Verantwortlichkeit. ${ }^{152}$ Spezielle Zurechnungsregeln bestehen deshalb auch nur im Völkerstrafrecht. ${ }^{153}$ Der Rückgriff auf Staaten als Zurechnungs- und Verantwortungssubjekt wird aus diesem Grund notwendig. Die Voraussetzungen dafür, wann ein Verhalten „Privater" dem Staat zurechenbar ist, sind zwar eng gefasst, ${ }^{154}$ jedoch bieten die den Staaten obliegenden Schutzpflichten grundsätzlich die Möglichkeit, den Gefährdungen durch „Private“ begegnen zu können, ${ }^{155}$ wenngleich bezüglich der bestehenden Schutzpflichten mehr Rechtssicherheit wünschenswert wäre. Zudem kann das Konzept völkerrechtlicher Verantwortlichkeit aufgrund der ihm zugrundeliegenden Trennung von privatem und staatlichen Verhalten das komplexe Zusammenspiel staatlicher und privater Kräfte nicht wiederspiegeln. ${ }^{156}$ Beispielsweise greifen die Regierungen schwacher Staaten auf private Militärfirmen zurück, um sich selbst gegen innerstaatliche Unruhen zu schützen und ihre Hoheitsgewalt durchzusetzen. ${ }^{157}$ Der Verweis auf die staatliche Verantwortlichkeit läuft ins Leere, wenn die Regierung für ihr Überleben auf private Sicherheitsfirmen angewiesen ist. ${ }^{158}$

Obwohl die bestehenden Zurechnungsregeln es ermöglichen, menschenrechtsverletzendes Verhalten internationalen Organisationen oder „Privaten“ zuzurechnen, ist, bedingt durch deren nur in sehr engem Rahmen bestehender Bindung an menschenrechtliche Standards und das Problem ihrer fehlenden Durchsetzbarkeit, der Rückgriff auf Staaten weiterhin notwendig, um Rechtsschutzlücken zu vermeiden.

\section{Jurisdiction als Anwendungsvoraussetzung menschenrechtlicher Verträge-Besteht Anpassungsbedarf?}

In einer Reihe menschenrechtlicher Verträge wird als Voraussetzung für die Anwendbarkeit der in ihnen garantierten menschenrechtlichen Vorschriften gefordert (Art. 1 EMRK, Art. 2 Übereinkommen über die Rechte des Kindes, Art. 1 Abs. 1 Amerikanischen Menschenrechtskonvention, Art. 2 Abs. 1 Internationaler Pakt über politische und bürgerliche Rechte und Übereinkommen gegen Folter und andere grausame, unmenschliche oder erniedrigende Behandlung oder Strafe, Art. 3 Internationales Ab-

152 Tomuschat, (Rn. 107), 274.

153 Safferling, (Fn. 51), § 5 Rn. 1.

154 siehe auch: Seibert-Fohr, (Fn. 126), 42.

155 Ibid., 60.

156 Lehnardt, (Fn. 26), 261.

157 Ibid., 20 ff., mit Beispielen: Angola, Sierra Leone, Papua-Neuguinea, Kroatien, Irak.

158 Ibid., 261. 
kommen zur Beseitigung aller Formen von Rassendiskriminierung), ${ }^{159}$ dass sich die betroffene Person unter der jurisdiction einer Vertragspartei befunden haben muss. ${ }^{160}$ Der englische Begriff jurisdiction und der französische Begriff der jurisdiction werden allgemeinhin weiter verstanden als die deutsche Übersetzung „Hoheitsgewalt“ oder „Herrschaftsgewalt“. ${ }^{161}$ Da die authentische Sprache der bestehenden menschenrechtlichen Verträge Englisch und/oder Französisch ist, eignet sich die missverständliche deutsche Übersetzung nicht als Grundlage für Auslegung und Betrachtung. ${ }^{162}$

\section{Die Ausübung von jurisdiction durch Staaten-Betrachtung der Rechtsprechung des EGMR}

Am Häufigsten hat sich der EGMR mit der Feststellung des Vorliegens oder des Nichtvorliegens von jurisdiction beschäftigt. Seine Rechtsprechung diesbezüglich hat großen Einfluss auf andere menschenrechtliche Kontrollinstanzen und den IGH, ${ }^{163}$ weshalb im Folgenden exemplarisch seine Rechtsprechung betrachtet werden soll.

Der Art. 1 EMRK, der den Anwendungsbereich der EMRK festlegt, sieht in seiner verbindlichen englischen bzw. französischen Sprachfassung vor:

"The High Contracting Parties shall secure to everyone within their jurisdiction the rights and freedoms defined in Section I of this Convention" bzw. "Les Hautes Parties contractantes reconnaissent à toute personne relevant de leur jurisdiction les droits et libertés définis au titre I de la présent Convention."

Eine verlässliche Begriffsinterpretation der jurisdiction lässt sich aus der Rechtsprechung der EGMR bislang nicht gewinnen. ${ }^{164}$ Im Schrifttum wird jurisdiction i.S.v. Art. 1 EMRK teilweise auf die Zurechnung einer Handlung zum Staat reduziert. ${ }^{165}$ Die Rechtsprechung des EGMR in der Rechtssache Bankovic, in der den Staaten die Bombardierung einer Radiostation zwar nach den Regeln der ASR zurechenbar war, aber der EGMR verneinte, dass diese jurisdiction über die Opfer ausgeübt haben, ${ }^{166}$ weist jedoch in eine andere Richtung. Sie stützt die Annahme, dass die Zurechnung den Beschwerdegegner bestimmt, das Innenverhältnis zwischen Staat und dem im konkreten

159 Jankowska-Gilberg, Extraterritorialität der Menschenrechte, Der Begriff der Jurisdiktion im Sinne von Art. 1 EMRK, 2008, 25.

160 Moos, (Fn. 18), 80-83; Holzinger, EMRK und internationale Organisationen, Die Zulässigkeit der Übertragung von Hoheitsrechten aus Sicht der EMRK und ihre Folgen für die konventionsrechtliche Verantwortlichkeit der Mitgliedstaaten 2010, 56; Gondek, The Reach of Human Rights in a Globalising World, 2009, 369.

161 Peters, (Fn. 4), 3.

162 siehe Art. 33 WVK.

163 Gondek, (Fn. 160), 371.

164 vgl. Moos, (Fn. 18), 81; Thallinger, (Fn. 78), 111 ff.; 179-203; Peters, (Fn. 4), 3 f.; Grundlegend Milanovic, From Compromise to Principle: Clarifying the Concept of State Jurisdiction in Human Rights Treaties, Human Rights Law Review 8, 2008, 411-448.

165 vgl. z.B. Schäfer, Der Fall Bankovic oder Wie eine Lücke geschaffen wird, MRM 7, 2002, $149,156$.

166 EGMR, Appl.No. 52207/99, Bankovic u.a. v. Belgien u.a., Urteil v. 12.12.2001, Rn. 82. 
Fall handelnden Akteur, ${ }^{167}$ während die jurisdiction das Verhältnis des Staates zum Beschwerdeführer, das Außenverhältnis zum Adressaten dieses Verhaltens, ${ }^{168}$ beschreibt. ${ }^{169}$ Nicht jeder Fall von Zurechnung eines Handelns zu einem Staat führt folglich dazu, dass auch das Vorliegen staatlicher jurisdiction zu bejahen ist. ${ }^{170}$

Art. 1 EMRK legt keinen örtlichen Anwendungsbereich der EMRK fest, weshalb sie grundsätzlich unabhängig von einer Begrenzung auf das Territorium der Vertragsstaaten Anwendung findet. ${ }^{171}$ Der EGMR unterscheidet bei seiner Prüfung, ob eine Person sich unter der jurisdiction eines Konventionsstaates befand, gleichwohl zwischen innerstaatlichen Handlungen der Konventionsstaaten und solchen, die sich außerhalb ihrer staatlichen Grenzen abspielen (extraterritoriales Handeln). Wenn eine innerstaatliche Handlung vorliegt, auch wenn diese Konsequenzen außerhalb des Staatsgebietes haben sollte, ${ }^{172}$ vermutet er, dass die sich auf dem Staatsgebiet des Konventionsstaates befindlichen Personen der jurisdiction des Staates unterstehen, ${ }^{173}$ woran auch die fehlenden Kontrolle über das eigene Staatsgebiet (Situation intra-territorial prekärer Hoheitsgewalt) ${ }^{174}$ nichts ändert. ${ }^{175}$ Auch Personen, die sich im Ausland befinden, unterliegen der jurisdiction eines Konventionsstaates, wenn dieser effektive Gesamtkontrolle (effective overall control) über ein bestimmtes Gebiet im Ausland und die sich dort befindlichen Individuen innehat, wobei es unerheblich ist, ob diese direkt durch Truppen des Staates oder indirekt durch untergeordnete lokale Verwaltungseinheiten erfolgt. ${ }^{176}$ Auch in diesem Fall wird vermutet, dass die sich dort befindlichen Personen der jurisdiction des Kontrolle ausübenden Konventionsstaates unterstehen. ${ }^{177}$ Zudem wird, bei fehlender Gebietskontrolle in Einzelfällen, wenn effektive Kontrolle (effective control) über bestimmte Personen ${ }^{178}$ oder Personen und Eigentum ${ }^{179}$ im Ausland durch den Staat ausgeübt wird, angenommen, dass die betroffene Person sich unter der jurisdiction des Staates befand. ${ }^{180}$ Nicht schon jede faktisch existierende Verbindung zwischen dem Betroffenen und dem Konventionsstaat reicht für die Begründung von jurisdic-

167 siehe bezogen auf Staaten, Moos, (Fn. 18), 81.

168 Thallinger, (Fn. 78), 152.

169 Jankowska-Gilberg, (Fn. 159), 135.

170 Thallinger, (Fn. 78), 152.

171 vgl. Jankowska-Gilberg, (Fn. 159), 127.

172 EGMR, Appl.No.14038/88, Soering v.Vereinigtes Königreich, Urteil v. 7.7.1989, Rn. 85 ff..

173 vgl. hierzu ausführlich Thallinger, (Fn. 78), den eine Auswertung der Rechtsprechung, Literatur und die Auslegung des Art. 1 EMRK zu diesem Schluss kommen lässt, 113-179, 134, 206; Moos, (Fn. 18), 90 f.; Peters, (Fn. 4), 31; s. auch EGMR, Appl.No.27765/09, Hirsi Jamaa u.a. v. Italien, Urteil v. 23.2.2013, Rn. 7. Weil nach den maßgebenden Regelungen des Seerechts ein Schiff auf hoher See ausschließlich der Hoheitsgewalt des Flaggenstaates untersteht, nimmt der EGMR bei Maßnahmen, die an Bord von Schiffen unter der Flagge eines Staates ebenso wie in dort registrierten Flugzeugen vorgenommen werden, die extraterritorialer Ausübung der Hoheitsgewalt dieses Staates an.

174 diese Begrifflichkeit verwendet Peters,(Fn. 4), 18.

175 EGMR, Appl. No.48787/99, Ilascu v. Moldawien/Russland, Urteil v. 8.6.2004, Rn. 333.

176 z.B. EGRM, Bankovic. u.a. v. Belgien u.a., (Fn. 166), Rn. 71.

177 Siehe auch Peters, (Fn. 4), 8.

178 EGMR, Appl. No. 46221/99, Öcalan v. Türkei, Urteil v. 12. 5.2005, Rn. 91.

179 EKMR, $X / Y$ v. Schweiz, Entscheidung v. 14.7.1977, 71.

180 vgl. hierzu ausführlich Thallinger, (Fn. 78), 113-179,134, 206; EGMR, Amuur v. Frankreich, Appl. No. 19776/91, Urteil v. 25.6.1996; Gondek, (Fn. 160), 371. 
tion aus, sondern es ist eine rechtliche Verbindung (jurisdictional link) notwendig, ${ }^{181}$ die gegeben ist, wenn ein Staat gegenüber einer konkreten Person rechtliche Anordnungen treffen und diese gegebenenfalls auch mit Zwang durchsetzen kann. ${ }^{182}$

Trotz der soeben aufgezeigten erkennbaren Rechtsprechungslinien, wird die Rechtsprechung des EGMR zum Vorliegen von jurisdiction allgemeinhin als einzelfallbezogen und inkonsistent empfunden. Zur Rechtsunsicherheit hat insbesondere die Bankovic-Entscheidung beigetragen, in der der EGMR den erforderlichen jurisdictional link durch den angefochtenen Akt der Bombardierung einer Radiostation außerhalb der Territorien der EMRK-Staaten nicht begründet sah. ${ }^{183}$ Diese restriktive Auslegung der Begriffs jurisdiction wurde vielfach als nicht mit seiner bis dato erfolgten Rechtsprechung vereinbar angesehen, ${ }^{184}$ da er in dieser das Konzept der Regionalität der EMRK neu einführte ${ }^{185}$ und zum ersten Mal erklärte, dass jurisdiction primär territorial beschränkt sei. ${ }^{186}$ Es sorgte im Schrifttum für wenig Verständnis, dass eine Person, die der Waffengewalt eines Staates ausgesetzt wird, nicht der jurisdiction dieses Staates unterstehen soll. ${ }^{187}$

Es stellt sich die Frage, ob die vom EGMR angenommene Unterscheidung zwischen Inland und Ausland hinsichtlich des Vorliegens von juridiction in Zeiten der Entterritorialisierung noch angebracht ist. Staaten werden nicht mehr nur auf ihrem eigenen Territorium tätig und üben ihre Staatsgewalt nur auf diesem aus. Zum anderen treffen überstaatliche Einheiten oder Institutionen Entscheidungen, welche auf dem Territorium der Staaten Geltung beanspruchen (Entstaatlichung). ${ }^{188}$ Staaten existieren selbst auf ihrem Staatsgebiet nicht mehr als einzige Staatsgewalt, die sich ihre Herrschaftsberechtigung mit niemand teilen muss. ${ }^{189}$

Ein Nachweis, dass das Opfer sich unter der jurisdiction eines Konventionsstaates befand, muss gegenwärtig nur außerhalb dessen Staatsgebietes erbracht werden. Entweder dergestalt, dass ein Staat effektive Gesamtkontrolle über ein fremdes Gebiet ausübt oder, dass im Einzelfall das Vorliegen eines jurisdictional link zwischen Opfer und Konventionsstaat bestand. Bei einer sich auf dem Territorium eines Staates befindlichen Person wird hingegen vermutet, dass sie der jurisdiction des Staates unterstand. Letzt-

181 Holzinger, (Fn. 160), 38; a.A. Schäfer, (Fn. 168), 156 bejaht zwar das Vorliegen eines rechtlichen Bandes, sieht dies aber auch bei faktischer Ausübung von Hoheitsgewalt gegeben.

182 Holzinger, (Fn. 160), 38.

183 EGMR, Bankovic u.a.v.Belgien u.a. (Rn. 166), Rn. 82.

184 siehe z.B. Roxstrom/Gibney/Einarsen, The Nato Bombing Case (Bankovic et al. v. Belgium et al.) and the Limits of Western Human Rights Protection, BUILJ 23, 2005, 56, $77 \mathrm{ff}$.

185 Jankowska-Gilberg, (Fn. 159), $143 \mathrm{ff}$.

186 EGMR, Bankovic u.a. v.Belgien u.a. (Rn. 166), Rn 59; Hirsi u.a. v. Italien, (Fn. 173) Rn. 7.

187 s. z.B. Sondervotum von Richter Loucaides und Richter Kovlar in: EGMR, Ilascu, (Fn. 175); a.A. Jankowska-Gilberg, (Fn. 159), 81 ff.; sieht den jurisdictional link (Kontrolle und Einflussmöglichkeit des Staates), zwischen Opfer und Staat nicht gegeben. Bedeutsam ist für sie, ob der Staat die EMRK gezielt verletzt. Dann könne er sich nicht der Prüfung durch die EMRK entziehen. Wenn sich ein Staat bei einem Luftangriff auf ein nicht besetztes Territorium aber an die durch das humanitäre Völkerrecht vorgegebenen Vorsichtsmaßnahmen halte, führe er die Betroffenen nicht unter seine jurisdiction.

188 Grimm, (Fn. 5), 156 ff.

189 Feinäugle, (Fn. 5), 23; Cassese, (Fn. 5), 663, 674. 
endlich ist also, je nach Ort des Geschehens, die Beweislast dafür, dass eine Person der jurisdiction eines Konventionsstaates unterstand, eine andere. Die oben beschriebene Entwicklung legt eine Vereinheitlichung der Regelungen für das Vorliegen von jurisdiction nahe. Erstgenannte Entwicklung spricht dafür, dass die Vermutung, dass eine Person der jurisdiction eines Staates untersteht, allgemeinhin und nicht nur für das Staatsgebiet, angenommen werden sollte. Während die zweitgenannte Entwicklung eher nahe legt, dass das Bestehen eines jurisdictional link zwischen Opfer einer Menschenrechtsverletzung und einem Staat in jedem Einzelfall erst festgestellt werden sollte, unabhängig vom Ort des Geschehens. Mit dem Argument, dass im Zeitalter der Globalisierung und der damit einhergehenden Relativierung territorialer Grenzen es wenig sachgemäß erscheine, die Verantwortung von EMRK-Vertragsstaaten untereinander anhand des Orts des konkreten Geschehens zu verteilen und somit das Entstehen von Rechtsschutzlücken zu riskieren, schlägt Peters (zudem) vor, die Vermutung des Vorliegens von jurisdiction nur für den espace jurisdique der EMRK anzunehmen. ${ }^{190}$

All diese Vorschläge können die komplexe Wirklichkeit jedoch nicht zufriedenstellend wiedergeben. Die Möglichkeit von Staaten, im Ausland rechtliche Anordnungen zu treffen und durchzusetzen, ist bislang nicht derart ausgeprägt, dass eine Vermutung zugunsten dieser Annahme angebracht erscheint. Trotz der Entwicklung der Entstaatlichung ist der jurisdictional link zwischen einer sich auf dem Territorium eines Staates befindlichen Person und dem Staat in der Regel weiterhin gegeben, weshalb diese auch weiterhin vermutet werden kann. Zweitgenannte Auffassung würde insoweit keine Unterschiede zur bestehenden Situation bedeuten, als auf dem eigenen Staatsgebiet der Beweis für das Vorliegen einer effektiven Gesamtkontrolle nicht schwer erbringbar ist. Eine Engführung des Menschenrechtsschutzes könnte sich allerdings insoweit ergeben, als es schwerer würde, Schutzpflichten eines Staates zu begründen. Das Argument der fehlenden Kontrolle über Teile des eigenen Staatsgebiets (Situation intra-territorial prekärer Hoheitsgewalt) müsste dann nämlich wohl gelten gelassen werden. Zu drittgenanntem Vorschlag ist zu bemerken, dass sich die Globalisierung und die Relativierung territorialer Grenzen unzweifelhaft nicht nur auf die EMRK-Staaten begrenzte Phänomene darstellen, weshalb es keinen erkennbaren Grund gibt, warum eine Begrenzung der Verantwortung der EMRK-Staaten außerhalb des EMRK-Gebietes angebracht ist und Rechtsschutzlücken insoweit hinzunehmen seien sollen.

\section{Die Ausübung von jurisdiction durch internationale Organisationen}

Fraglich ist, inwieweit sich die vom EGMR aufgestellten Regelungen dafür, wann eine Person der jurisdiction eines Staates unterliegt, zur Anwendung auf internationale Organisationen eignen, in Anbetracht dessen, dass die Anwendungs-und Durchsetzungsbefugnis auf der Völkerrechtsebene deutlich schwächer ausgebildet ist als auf nationaler Ebene $^{191}$ und internationale Organisationen über kein Staatsgebiet verfügen.

Die CDDH hat den Vorschlag unterbreitet, dass bezogen auf die EU nach deren Beitritt zur EMRK folgendes gelten soll:

190 Ibid., $42 \mathrm{ff}$.

191 Statt vieler Dahm/Delbrück/Wolfrum, (Fn. 13), § 8, 88 ff. 
"Insofar as the expression "everyone within their jurisdiction" appearing in Article 1 of the Convention refers to persons within the territory of a High Contracting Party, it shall be understood, with regard to the European Union, as referring to persons within the territories of the member States of the European Union to which the Treaty on European Union and the Treaty on the Functioning of the European Union apply. Insofar as this expression refers to persons outside the territory of a High Contracting Party, it shall be understood, with regard to the European Union, as referring to persons who, if the alleged violation in question had been attributable to a High Contracting Party which is a State, would have been within the jurisdiction of that High Contracting Party." 192

Die Orientierung an der EGMR-Rechtsprechung ist klar erkennbar. Wird der CDDHVorschlag angenommen, müsste der EGMR deshalb von seiner bisherigen Rechtsprechung zum Vorliegen von jurisdiction, bezogen auf die Vertragspartei EU, nicht abweichen. Im „Inland“, dem gesamten Territorium der EU-Mitgliedstaaten, würde die Vermutung gelten, dass diese jurisdiction über die sich auf diesem Gebiet befindlichen Personen besitzt. Nicht relevant für die CDDH ist augenscheinlich, dass die EU kein Staatsgebiet besitzt, über das sie die vollständige jurisdiction innehat, sondern, wie alle internationale Organisationen, nur begrenzte funktionale jurisdiction besitzt. ${ }^{193}$ Vielmehr ist für sie ausschlaggebend, dass auf dem Territorium der EU-Mitgliedstaaten die primärrechtlichen Verträge der EU Anwendung finden, die Anordnungs- und Durchsetzungsbefugnis der EU auf diesem Gebiet also normalerweise zur Geltung kommt. Die EU nimmt jedoch insoweit eine Sonderrolle ein, als sie als supranationale Organisation ausgeprägtere Bindungs- und Zwangsmöglichkeiten (bzw. Anordnung und Durchsetzungsbefugnisse) als andere internationale Organisationen hat. Sie kann Recht setzen, das unmittelbar in den Mitgliedstaaten wirkt, unmittelbare Wirkung auch gegenüber Einzelnen erlangt und (Anwendungs-) Vorrang vor nationalem Recht beansprucht. Dennoch ist zu monieren, dass ihre Anordnungs- und Durchsetzungsbefugnisse im Vergleich zu Staaten beschränkt sind.

Dass die Organe der EU in nächster Zeit selbst zivile oder militärische Missionen außerhalb des Territoriums ihrer Mitgliedstaaten durch eine europäische Armee ausführen und ihr somit das Handeln der Angehörigen der Streitkräfte einer EU-Mission nach den CDDH-Regeln zurechenbar wäre, ist in naher Zukunft nicht zu erwarten. Das Verhalten mitgliedstaatlicher Truppen, also staatlichen Organen, in einer von der EU geführten Mission, wäre dem CDDH-Vorschlag folgend, somit immer den Mitgliedstaaten zurechenbar, weshalb die EU für dieses auch nach ihrem Beitritt zur EMRK vor dem EGMR nicht zu Verantwortung gezogen werden könnte. Trotz fehlender Zurechnung des Verhaltens der mitgliedstaatlichen Truppen zur EU ist es jedoch möglich, dass die EU jurisdiction über eine sich im Ausland befindliche Person ausübt. ${ }^{194}$ Insbesondere ist es möglich, das der handelnde Mitgliedstaat allein keine wirksame territoriale Gesamtkontrolle über ein bestimmtes Gebiet im Ausland hat, aber die EU diese Kontrolle ausübt, ${ }^{195}$ wenn man die sich aus verschiedenen nationalen Truppenkontigenten

192 Final Report of the CDDH, (Fn. 35), Art. 1, 5, Rn. 6.

193 s. auch Naert, International Law Aspects of the EU's Security and Defence Policy, with a particular focus on the law of armed conflict and human rights, 2008, 424.

194 so allgemein auch Holzinger, (Fn. 160), 41.

195 vgl. hierzu ausführlich Thallinger, (Fn. 78),113-179; Moos, (Fn. 18), 90 f. 
zusammensetzende Armee, von denen jedes für sich betrachtet, nur einen Teil der effektiven Kontrolle ausübt, ${ }^{196}$ hierfür als Einheit betrachtet. Das Vorliegen von jurisdiction über dieses Gebiet würde vermutet, was den nicht immer leicht zu erbringenden Beweis für das Vorliegen eines jurisdictional link zwischen Opfer und Konventionsstaat obsolet machen würde. ${ }^{197}$ Ansonsten bestünde die Möglichkeit, ein Verhalten, das bei Anwendbarkeit der Regeln der DARIO (Art. 7 DARIO, Organleihe) der EU zurechenbar wäre und bei der das Opfer, durch die von der EU ausgeübte effektive Gesamtkontrolle, auch der jurisdiction der EU unterlegen hätte, folglich die EMRK anwendbar wäre, aufgrund der von der CDDH vorgesehenen formalen Zurechnungsregeln nicht zur Anwendbarkeit der EMRK führen würde. Der von der CDDH vorgeschlagene Mitbeklagten-Mechanismus würde in einem solchen Fall nicht helfen, da die Zulässigkeit einer Klage unabhängig davon beurteilt werden soll, ob ein Mitbeklagter am Prozess teilnimmt. ${ }^{198}$ Dieses unbefriedigende Ergebnis kann dadurch vermieden werden, dass Art. 1 EMRK dahingehend ausgelegt wird, dass die Hohe Vertragspartei jurisdiction nicht zwingend selbst ausüben muss, ${ }^{199}$ sondern es ausreicht, wenn die EU dem Opfer gegenüber eigene jurisdiction ausübt. ${ }^{200}$ Zwar nimmt der EGMR in der Behrami/Saramati-Entscheidung keine explizite Stellung dazu, ob dies möglich ist, jedoch kommt er zu dem Schluss, dass der Kosovo unter der „effektiven Kontrolle der internationalen Präsenz" stehe, und dass die Vereinten Nationen ,overall effective control “ über das Gebiet ausübten, ${ }^{201}$ prüfte in einem zweiten Schritt jedoch noch, ob die Handlungen den Mitgliedstaaten oder den Vereinten Nationen zurechenbar sind. ${ }^{202}$ Dies deutete darauf hin, dass die jurisdiction nicht zwingend von Mitgliedstaaten selbst ausgeübt werden muss. Ansonsten hätte dem EGMR nämlich schon der Befund, dass die Vereinten Nationen ,overall effective control “ über das Gebiet ausübt ausreichen müssen, um seine Unzuständigkeit feststellen zu können, bzw. er hätte schon an dieser Stelle prüfen müssen, ob der jurisdictional link zwischen den Opfern und den Konventionsstaaten bestand. ${ }^{203}$ Auch der Wortlaut des Art. 1 EMRK stünde einer solchen Auslegung nicht entgegen, da er nur vorgibt, dass die betroffene Person der jurisdiction einer Vertrags-

196 Moos, (Fn. 18), 92.

197 siehe z.B. EGMR, Appl. No. 31821/96, Issa u.a v.Türkei, Urteil v. 16.11.2004, Rn. 76 ff. in der der Beweis dafür, dass sich die Streitkräfte der Türkei auf dem Gebiet aufgehalten haben, in dem die beklagte Tötung stattfand, nicht erbracht werden konnte.

198 Final Report of the CDDH, (Fn. 35), Art. 3, 6, Rn. 1.

199 so verlangt es aber z.B. Matscher, Das Verfahren vor den Organen der EMRK, EuGRZ $1982,489,500 \mathrm{ff}$.

200 Holzinger, (Fn. 163), 41.

201 EGMR, Behrami und Saramati v. Frankreich u.a. (Fn. 96), Rn. 70. Weiter wies es darauf hin, dass die internationale Präsenz ,die öffentlichen Befugnisse ausübte, die normalerweise von der Regierung der FRY ausgeübt würden“" sowie auf seine Bankovic-Rechtsprechung und knüpfte somit an seine Rechtsprechung zur extraterritorialen Anwendbarkeit der EMRK an. Unmittelbar im Anschluss stellte er aber fest, dass es hier weniger auf die Extraterritorialität als auf seine gerichtliche Überprüfungszuständigkeit ankomme (Rn. 71).

202 Ibid., Rn. 71 und Rn. 128 ff.

203 Er verzichtet darauf zu prüfen, ob die Konventionsstaaten zumindest jurisdiction über die betroffenen Personen ausübten. Fraglich ist, ob er diese Prüfung vorgenommen hätte, wenn das Verhalten den Konventionsstaaten zurechenbar gewesen wäre; Ibid., Rn. 153. 
partei unterstehen muss, hingegen nicht, dass im konkreten Fall die Vertragspartei diese selbst ausgeübt haben muss. ${ }^{204}$

Bei anderen internationalen Organisationen ist es bei Anwendung der Regeln der DARIO auch heute schon möglich, dass ihnen Verhalten mitgliedstaatlicher Truppen zurechenbar ist. Bei Anwendung der CDDH-Vorschlages und der EGMR-Rechtsprechung könnte Ergebnis sein, dass bei einer Peacekeeping-Mission der Vereinten Nationen in einem seiner 193 Mitgliedstaaten die Vermutung für die Ausübung von jurisdiction bestehen würde, während, wenn das Verhalten mitgliedstaatlicher Truppen den Mitgliedstaaten selbst zurechenbar wäre, das Vorliegen von jurisdiction außerhalb des eigenen Staatsgebietes erst begründet werden müsste. Ein unbefriedigendes Ergebnis, in Anbetracht der sehr begrenzten Anordnungs- und Durchsetzungsbefugnis internationaler Organisationen auch auf dem Territorium ihrer Mitgliedstaaten.

\section{Zwischenfazit}

Das bestehende Verständnis von jurisdiction ist staatenzentriert. Grundannahme ist, dass ein Staat seine jurisdiction grundsätzlich auf seinem Staatsgebiet ausübt und deshalb die sich auf diesem Territorium befindlichen Personen seiner jurisdiction unterworfen sind. Diese Grundannahme wird jedoch von vielen Seiten in Frage gestellt. Staaten werden zunehmend auch außerhalb ihres Staatsgebietes tätig und üben auf diesem nicht mehr die umfassende und einzige Hoheitsgewalt aus. Die bestehende Rechtsprechung zum Vorliegen von jurisdiction gibt diese Entwicklungen nicht wieder. Zumindest für die EU als internationalen Organisation schlägt die CDDH vor, diese im Ergebnis gleich einem Staat zu behandeln bei der Beantwortung der Frage, ob ein Opfer ihrer jurisdiction unterlag, ohne auf bestehende Unterschiede, vor allem ihre nur begrenzten funktionalen jurisdiction einzugehen. Ausschlaggebend ist für sie für die Vermutung des Vorliegens von jurisdiction, dass die Bindungs- und Zwangsmöglichkeiten der EU auf dem Gebiet ihrer Mitgliedstaaten normalerweise zur Geltung kommen.

Die heute vorgegebene Alles-oder-Nichts-Entscheidung, die bezüglich des Eintretens auf eine Beschwerde oder nicht gegeben ist, ${ }^{205}$ kann die aufgezeigten Entwicklungen nicht befriedigend wiedergeben. Daran würde sich, wie aufgezeigt, nichts ändern, wenn entweder (nur) vermutet würde, dass die Hohen Vertragsparteien der EMRK grundsätzlich auch außerhalb ihres Territoriums jurisdiction ausüben oder die Vermutung der Ausübung von jurisdiction über die sich auf dem Gebiet einer Vertragsparteien befindlichen Personen nicht mehr gelten würde.

\section{Flexiblere Lösungsansätze}

Im Schrifttum existieren verschiedene Konzepte, die Fragen der Ausübung von Hoheitsgewalt bzw. jurisdiction auf völkerrechtlicher Ebene behandeln. Zu nennen sind

204 vgl. Grabenwarter, Europäisches und nationales Verfassungsrecht, VVDStRL 60, 2001, 290, 331; Schäfer, Verletzungen der EMRK durch Europäisches Gemeinschaftsrecht und dessen Vollzug, 2006, 183; Holzinger, (Fn. 160), 40.

205 Peters,(Fn. 4), 45. 
das Konzept der global governance, des Global Administrative Law, des internationalen Verwaltungsrechts, der Konstitutionalisierung des Völkerrechts, sowie der öffentlichrechtliche Ansatz. ${ }^{206}$ Während einige dieser Konzepte sich eher darauf beschränken, beobachtete Phänomene zu beschreiben und zu verstehen (z.B. das global governance Konzept), ${ }^{207}$ existieren auch umfassende Ansätze, die sich allgemein damit beschäftigen, wie sich die Ausübung von ,völkerrechtlicher Hoheitsgewalt“ rechtlich am besten fassen lässt, wobei Legitimations- und Schrankenfragen, auch die nach der Beschränkung durch menschenrechtliche Standards, im Vordergrund stehen (öffentlich-rechtlicher Ansatz). ${ }^{208}$ Eine Besprechung dieser Konzepte ist im Rahmen dieses Beitrages nicht möglich. Es lässt sich jedoch ausmachen, dass ihnen, trotz bestehender Unterschiede, gemein ist, dass sie versuchen, die Besonderheiten der Völkerrechtsordnung zu erfassen, um das Phänomen der Ausübung von Hoheitsgewalt auf völkerrechtlicher Ebene rechtlich fassen zu können. Beispiele für Regeln der Völkerrechtsordnung sind der Grundsatz der Immunität internationaler Organisationen vor nationalen Gerichten, der einer einfachen Übertragung nationaler Vorstellungen effektiven Rechtsschutzes entgegenstehen könnte, die eingeschränkte Durchsetzbarkeit des Völkerrechts, die besondere Bedeutung politischer Interessen, der Mangel an Vorrang, Hierarchie und Supranationalität, der die Völkerrechtsordnung kennzeichnet, der Aspekt des Handelns ultra vires durch internationale Organisationen, sowie die Mehrebenenarchitektur, die bei der Ausübung von Hoheitsgewalt auf völkerrechtlicher Ebene typischerweise vorzufinden ist. ${ }^{209}$ Verdeutlicht wird, der Bedarf nach flexiblen Lösungen, die die Beachtung der Besonderheiten der Völkerrechtsordnung bei der rechtlichen Fassung von Hoheitsgewalt im Einzelfall gewährleisten können.

Fraglich ist, wie sich diese Erkenntnisse im Rahmen einer Klage vor dem EGMR oder anderer menschenrechtlicher Kontrollinstanzen fruchtbar machen lassen. Eine differenzierte Lösung wäre, wie Peters es vorschlägt, auf der materiell rechtlichen Ebene (Schutzbereichseröffnung und Einschränkbarkeit) zu erreichen. ${ }^{210}$ Um diese materielle Abwägung vornehmen zu können, ist vorzuschlagen, zunächst das Vorliegen von jurisdiction allgemeinhin zu vermuten und somit die Klage für zulässig zu erklären. ${ }^{211}$ Auf die Entwicklung von komplexer Hoheitsgewalt und Entterritorialisierung könnte sodann durch eine auf die Doktrin der margine d'appréciation gestützte Abstufung von Menschenrechtsverletzungen reagiert werden. ${ }^{212}$ Im Einzelfall wäre zu ermitteln, ob und welche Verpflichtungen der Vertragspartei bestehen oder ob diese eine übermäßige Belastung (undue burden) für diese darstellt. ${ }^{213}$ Im Ausland ist beispielsweise das Recht auf Leben eher anwendbar als das Recht auf Asyl, der status negativus leichter und problemloser zu gewähren und für die Gewährung einiger Verfahrensgarantien müssten

206 Feinäugle, (Fn. 5), 52 ff.

207 Ibid., 54.

208 Ibid., $46 \mathrm{ff}$.

209 Ibid., $68 \mathrm{f}$.

210 siehe Peters, (Fn. 4), 45.

211 a. A. Ibid., 45-52; Die Frage nach der Jurisdiktion sei dogmatisch gesehen keinen Zulässigkeitsaspekt, sondern eine materiellrechtliche Frage. Zudem möchte sie die Vermutung des Vorliegens von jurisdiction nur für den espace juridique der EMRK annehmen.

212 Ibid., 52.

213 Ibid. 
Institutionen vor Ort verfügbar sein, weshalb die Konventionsparteien insoweit an ihre Leistungsgrenzen geraten können. ${ }^{214}$

Vorteil der Flexibilität wäre auch, dass Grenzen, die der Staatstätigkeit durch das allgemeine Völkerrecht gesetzt werden, bei der Bestimmung der Grundrechtsverpflichtung Beachtung finden können. ${ }^{215}$ In Besprechungen der Bankovic-Entscheidung haben sowohl diejenigen, die sich gegen und diejenigen, die sich für die Zulässigkeit der Beschwerde bei einem solchen oder ähnlich gelagertem Sachverhalt ausgesprochen haben, die Notwendigkeit gesehen, dass das allgemeine Völkerrecht und insbesondere das humanitäre Völkerrecht bei der Entscheidung über die Konventionswidrigkeit der beanstandeten Handlung beachtet werden muss. ${ }^{216}$ Ausgehend von der Annahme, dass Menschenrechtsverträge auch in bewaffneten Konflikten fortgelten und die beiden Rechtsbereiche des humanitären und der Menschenrechte grundsätzlich kumulativ Anwendung finden, ${ }^{217}$ können die spezielleren Normen der kriegsrechtlichen Regelung zur Ergänzung und Konkretisierung der allgemeinen Menschenrechte herangezogen werden. ${ }^{218}$ Folge ist, dass ein mit dem humanitären Völkerrecht konformes Handeln, als lex specialis gegenüber dem menschenrechtlichen Vertrag, nicht dazu führten kann, dass diese als Verletzung einer menschenrechtlichen Bestimmung angesehen wird. ${ }^{219}$ Zudem kann sichergestellt werden, dass Regelungsansprüche anderer Staaten und Regime nicht verletzt werden, was insbesondere zu Problemen führt, wenn die Anwendung von Grundrechten im Ausland die Vornahme von extraterritorialen Vollstreckungshandlungen impliziert. 220

\section{Fazit}

Thürer hält es nicht für möglich, die Herausforderungen des Menschenrechtsschutzes, die sich gegenwärtig stellen, eine nach dem anderen in den Griff zu bekommen und für immer zu bewältigen. Menschenrechte seien immer die Antwort auf konkrete Leiden und Exzesse der Macht im Laufe der Geschichte. ${ }^{221}$

Als gegenwärtige große Herausforderungen lassen sich unter anderem die Verletzung menschenrechtlich geschützter Inhalte durch nichtstaatliche Akteure, die Komplexität der ausgeübten Hoheitsgewalt (jurisdiction) und die voranschreitende Entterritorialisierung, bei gleichzeitig weiterhin bestehender Staatenzentriertheit des Menschenrechtsregimes, ausmachen. Die Staatenzentriertheit des Menschenrechtsschutzes ist darin zu sehen, dass Menschenrechtsregime weiterhin darauf ausgerichtet sind, Hoheitsgewalt im ,,herkömmlichen“ staatenrechtlichen Sinn zu begrenzen. Dieser Zustand führt

214 Ibid., 1 und 53.

215 Ibid.

216 Jankowska- Gilberg, (Fn. 159), 82.

217 Ibid., 85.

218 Kunig/Uerpmann-Wittzack, Übung im Völkerrecht, 2. Aufl., 2006, 207; Jankowska- Gilberg, (Fn. 159), 85.

219 Eick, Die Anwendbarkeit des IPBPR bei Auslandseinsätzen der Bundeswehr, in: Dupuy et al. (Hrsg.),Völkerrecht als Werteordnung, FS Tomuschat, 2006, 126.

220 Peters, (Fn. 4), 54.

221 Thürer, (Fn. 21), 586. 
dazu, dass der Begrenzung von (Hoheits)gewalt durch die Menschenrechte Grenzen gesetzt werden.

Dem Handeln „Privater“, das menschenrechtliche Inhalte verletzt, werden zum großen Teil dadurch Grenzen gesetzt, als dass dieses die Verantwortlichkeit von Staaten zur Folge haben kann. Auch wenn die grundsätzliche Trennung von privatem und staatlichem Verhalten das komplexe Zusammenspiel staatlicher und privater Kräfte nicht immer wiederspiegeln kann, ${ }^{222}$ wird allgemeinhin kein Bedarf dafür gesehen, „Private“ umfassend direkt an menschenrechtliche Standards zu binden.

Hingegen wird für internationale Organisationen der Bedarf nach einer umfassenden Bindung an menschenrechtliche Standards gesehen. Selbst wenn man jedoch über die allgemeinen Rechtsgrundsätze eine umfassende Bindung internationale Organisationen an menschenrechtliche Standards begründen kann, fehlt diesen bislang aber das Bewusstsein für diese Bindung und es fehlt an Möglichkeiten der Durchsetzbarkeit der gewährten Rechte. Bislang ist mit der EU nur eine internationale Organisation an einen speziellen menschenrechtlichen Vertrag (UN-Behindertenrechtskonvention) gebunden. Eine Grenze der Begrenzung von (Hoheits)gewalt kann sich insoweit ergeben, als dass Folge der Zurechnung einer menschenrechtsverletzenden Handlung zu einer internationalen Organisation gegenwärtig sein kann, dass das Opfer überhaupt keinen Rechtsschutz durch eine menschenrechtliche Kontrollinstanz erlangen kann (EGMR Behrami/ Saramati), da die Mitgliedstaaten der internationalen Organisation für diese Verletzung nicht verantwortlich gemacht werden.

Eine Grenze der Begrenzung von (Hoheits)gewalt ergibt sich auch aus der bestehenden Rechtsprechung menschenrechtlicher Kontrollinstanzen zur Erfüllung der Voraussetzung vieler menschenrechtlicher Verträge, dass das potenzielle Opfer der jurisdiction einer Vertragspartei unterstanden haben muss. Ausgangspunkt ist ein staatenzentriertes Verständnis von jurisdiction. Kritisieren lässt sich, dass die gegenwärtige Rechtsprechung des EGMR, (die großen Einfluss auf andere menschenrechtliche Kontrollinstanzen hat ${ }^{223}$ wonach auf dem eigenen Staatsgebiet eines Konventionsstaates vermutet wird, dass jurisdiction über eine sich auf diesem befindliche Person vorliegt, während auf fremden Territorium entweder festgestellt werden muss, dass der handelnde Staat effektive Gesamtkontrolle über ein Gebiet oder effektive Kontrolle über eine Person oder Sache hatte, nicht mehr den Gegebenheiten der Wirklichkeit entspricht. Es wird weder dem Fakt Rechnung getragen, dass Staaten zunehmend außerhalb ihres Territoriums handeln, noch dem Fakt, dass Staaten selbst auf ihrem Staatsgebiet nicht mehr als einzige Staatsgewalt, die sich ihre Herrschaftsberechtigung mit niemand teilen muss, existieren. ${ }^{224}$ Wendet man die Rechtsprechung des EGMR, wie es der CDDHVorschlag nahe legt, auch auf internationale Organisationen an, wird nicht beachtet werden, dass die Anordnungs- und Durchsetzungsbefugnisse auf völkerrechtlicher Ebene deutlich schwächer ausgeprägt sind als auf nationaler Ebene.

Differenzierte Ergebnisse sind auf Ebene der Zulässigkeit einer Klage nicht möglich. Der hier gemachte Vorschlag, allgemein zu vermuten, dass jurisdiction vorliegt, jedoch dann auf materieller Ebene die Grundrechtsbindung gegebenenfalls zu modifizieren,

222 Lehnhardt, (Fn. 28), 261.

223 Gondek, (Fn. 160), 371.

224 vgl. Feinäugle, (Fn. 5), 23; so auch Cassese, (Fn. 5), 674. 
würde es hingegen ermöglichen, flexibler auf beschriebene Entwicklungen reagieren zu können. Der EGMR erklärte jedoch in seiner Bankovic-Entscheidung, dass die Verpflichtung aus Art. 1 EMRK, die Konventionsrechte zu sichern, nicht teilbar sei, also die Verpflichtungen aus der EMRK entweder als Ganzes oder gar nicht gelten. ${ }^{225} \mathrm{Er}$ lehnt eine „materielle Lösung“ also bislang ab.

Solange für die neuen Phänomene der Ausübung von (Hoheits)gewalt auf völkerrechtlicher Ebene keine funktionsfähige Völkerrechtsordnung zur Verfügung steht, in dem Sinne, dass sie in der Lage ist, diese durch menschenrechtliche Standards wirksam zu begrenzen, ist dafür zu plädieren, weiterhin staatenzentriert zu denken in dem Sinne, dass Staaten auch für das Handeln , ihrer“ internationalen Organisation zur Verantwortung gezogen werden können und weitreichend ihre Verantwortung durch das menschenrechtsverletzende Verhalten von „Privaten“ ausgelöst wird.

Komplexe Hoheitsgewaltsausübung erfordert komplexe Antworten. Die Völkerrechtsordnung befindet sich momentan in einer Übergangsphase. Die beschriebenen Herausforderungen wurden erkannt, befriedigende Lösungen wurden bislang jedoch noch nicht gefunden. Es handele sich bei den sich für den Menschenrechtsschutz stellenden Aufgaben, wie Thürer es zutreffend formulierte, um ,komplexe Aufgaben, mit denen sich Viele in langwieriger Arbeit, mit Geduld und Imagination zu befassen haben ". ${ }^{226}$ Wie die Antwort der Völkerrechtsordnung auch im Detail aussehen mag, Flexibilität und Differenzierung sind notwendig in Zeiten, in denen das starre Konzept staatlicher Hoheitsgewalt als Ausgangspunkt für die Begrenzung von Gewalt gegen Individuen nicht mehr der Wirklichkeit entspricht.

225 Jankowska-Gilberg, (Fn. 159), 153.

226 Thürer, (Fn. 21), 586. 